\title{
Estadísticas de mortalidad en México: muertes registradas en el año 2001
}

D e acuerdo con las cifras del Instituto Nacional de Estadística, Geografía e Informática (INEGI), en 2001 se registraron en México 443127 defunciones, de las cuales 2123 corresponden a mexicanos que residían en el extranjero y las que se excluyen del total para fines del análisis que a continuación se presenta.

De las 441004 defunciones registradas en las 32 entidades federativas (5 521 defunciones más que el año anterior), $44.5 \%$ correspondieron a mujeres y $55.5 \%$ a hombres. Se mantiene la tendencia decreciente de las muertes en los niños menores de cinco años de edad: en 2001, 96 de cada 1000 muertes ocurrieron en esa edad, aunque conviene observar que la variación estatal va desde 150 a 60, si no se consideran aquellas entidades en donde es alto el subregistro de muertes infantiles. Por otro lado, más de la mitad de las defunciones suceden después de los 68 años de edad en las mujeres $y$, después de los 60, en los hombres. En los cuadros II y III se presentan las variaciones de la edad de muerte entre las entidades federativas. Es importante mencionar que en algunos cuadros, además de la tasa cruda de mortalidad, se incluye la tasa estandarizada por edad. La razón es que esta última permite hacer comparaciones entre entidades federativas a pesar de que presentan diferente estructura de edades en la población. Para facilitar las comparaciones internacionales se decidió usar como población estándar la que recomienda la Organización Mundial de la Salud. ${ }^{1}$ (Ver nota metodológica).

En los cuadros V y VI se muestran las principales causas de mortalidad registradas en 2001 para mujeres y hombres a escala nacional. Se repite el mismo formato que se usó para el año 2000, es decir, las principales causas se presentan en un nivel de desagregación que incluso permite asociarlas con intervenciones en salud específicas, evitando las agrupaciones en la medida de lo posible. En este sentido, destaca la diabetes mellitus que ocupa la primera causa de muerte en las mujeres $(14 \%)$ y la segunda en los hombres $(11 \%)$, seguida de la cardiopatía isquémica y de las enfermedades cerebrovasculares, que en números absolutos y en tasa todas aumentan. Además, llama la atención que el orden se modificó ligeramente en los hombres (cuadro VI) por la dismi- nución de las defunciones por accidentes de tráfico y por agresiones (homicidios).

Los cuadros VIII al XII incluyen las defunciones y las tasas estandarizadas, para mujeres y hombres por entidad federativa, de algunas causas de muerte seleccionadas por su importante magnitud.

En los cuadros XIII y XIV se presentan indicadores seleccionados sobre la calidad de la información de la mortalidad. En la primera columna del cuadro XIII se muestra el porcentaje de muertes que ocurrieron antes del año 2000, pero que fueron registradas ese año y el INEGI las reporta en su estadística. En el ámbito nacional se registran alrededor de 7500 defunciones que ocurrieron en años anteriores, observándose que en algunas entidades el registro extemporáneo asciende hasta $11 \% .^{2}$

Tradicionalmente se ha considerado que el certificado de defunción debe ser llenado por el médico y en México 98\% de ellos cumplen con este requisito. Lo más recomendable es que sea el médico que trató al paciente el que extienda el certificado de defunción, sin embargo, eso no sucede con la frecuencia esperada en

* Sección preparada por la Dirección General de Información y Evaluación del Desempeño. Secretaría de Salud. México. 
nuestro país. Si se descuentan las defunciones que por ley deben ser certificadas por los médicos legistas (defunciones por causas externas que son aproximadamente $52 \mathrm{mil}$ ) sólo $32 \%$ de las defunciones son certificadas por el médico tratante y el resto las certifica otro médico que no conocía a la persona que falleció. Cabe destacar que en el año 2000 las defunciones certificadas por el médico tratante ascendían a 34\% (de 131 mil bajó a 126 mil). De la misma manera se utiliza el indicador de las defunciones que sucedieron en las unidades médicas, en las que se presupone que los certificados llenados en dichos lugares son de mejor calidad (cuadro XIII).

Otro criterio de calidad es la identificación de las variables de la persona que falleció, por ejemplo, la edad o la condición de derechohabiencia. En México, en 2156 defunciones de mayores de un año de edad no se especifica la edad, ni tampoco en 530 que sucedieron antes de cumplir el año de edad. Actualmente uno de cada 11 certificados no especifica la variable derechohabiencia, sin embargo, en algunas entidades la relación asciende a uno de cada cinco.

La sección médica del certificado de defunción, y la correcta definición de la causa de muerte son fundamentales. Conviene insistir en la importancia que tiene el médico al momento de escribir en el certificado de defunción la secuencia de los padecimientos que antecedieron a la muerte, con el fin de que el codificador pueda aplicar las reglas de codificación y el principio general que aparece en la Clasificación Internacional de Enfermedades (CIE).
Desafortunadamente en nuestro país todavía existen problemas en el llenado del certificado y sus repercusiones van en detrimento de la calidad de las estadísticas de mortalidad.

En el cuadro XIV se presentan cinco indicadores que permiten evaluar la calidad de los certificados de defunción, en relación con la causa básica de muerte.

Del total de defunciones 2.1\% son clasificadas como Signos y Síntomas Mal Definidos (CIE 10 R00-99), aunque en algunas entidades ascienden a $5 \%$. Llama la atención que, en términos absolutos, las defunciones cuya causa estuvo mal definida ascendió de 8551 a 9195 . Otro criterio para medir la calidad de la causa de muerte se refiere a la precisión con la que una defunción por causa externa puede ser clasificada como intencional o accidental. La CIE-10 previene esta situación y establece un grupo de códigos para "eventos de intención no determinada" (Y10-Y34). En 2001 en México casi $4.5 \%$ de las 52 mil muertes por causas externas no pueden ser clasificadas como accidentales o intencionales, y caen en este rubro. Lo mismo sucede con las defunciones por tumores malignos de sitio no especificado.

Uno de los problemas típicos en la certificación de la muerte consiste en referir el mecanismo de la muerte y no la causa o enfermedad que la desencadenó. Los mejores ejemplos en este sentido son las muertes por insuficiencia cardiaca (CIE-10 I50) que pueden tener un origen isquémico, reumático, asociarse a una cardiopatía hipertensiva o con una cardiopatía por enfermedad de Cha- gas; o la insuficiencia hepática (CIE 10 K72) que, en su caso, puede estar relacionada con cirrosis hepática, hepatitis crónica o, incluso, hepatoma. Las últimas dos columnas del cuadro XIV dan cuenta de este problema al cual se podrían sumar la insuficiencia renal, la insuficiencia respiratoria y la ateroesclerosis inespecífica, entre otras. Lo paradójico del caso es que en aquellas entidades federativas en donde la proporción de insuficiencia cardiaca es alta (Tlaxcala, Oaxaca, Puebla) la mortalidad por cardiopatía isquémica es baja, quedando la duda acerca de si la baja mortalidad es real o se asocia con la mala clasificación de las defunciones.

En comparación con las defunciones registradas en el año 2000 se observan cambios en la estructura de causas y por edades, en la calidad y en la integralidad de los datos. Aún se detectan problemas de subregistro en algunas entidades federativas, así como problemas en el manejo de causas de muerte, lo que exige mantener los programas de capacitación para los médicos respecto al llenado del certificado defunción, y con los codificadores, para aplicar las actualizaciones de la Clasificación Internacional de Enfermedades.

REFERENCIAS

1.W orld Health 0 rganization. A ge standarization of rates: A new W HO standard. Ginebra:W HO, 2000; G PE D iscusion Paper Series N 0.31.

2. Para más detalle de las implicaciones que tiene el registro extemporáneo de las defunciones cf. Secretaría de Salud. Dirección General de Información y Evaluación del Desempeño. Tendencia de la mortalidad en México 1990-2000. Muertes ocurridas vs muertes registradas. En prensa. 


\section{Nota metodológica}

Uno de los procedimientos utilizados en el cálculo y presentación de tasas es el de estandarización. Con ello se controla el efecto de la variación en la estructura de edades de una población, y tiene la finalidad de elaborar indicadores resumen, comparables en diferentes contextos o para analizar su evolución en el tiempo.

El procedimiento de estandarización consiste en calcular una tasa resumen a partir de la suma ponderada de las tasas observadas por grupo de edad de la población de estudio.

Los ponderadores utilizados se corresponden con los valores derivados de la distribución porcentual por grupos de edad de la población tipo. Dado que la selección de la población tipo es arbitraria, en el caso que nos ocupa se consideró utilizar, para efectos de comparación internacional, la estructura reportada por la Organización Mundial de la Salud, que es el resultado de promediar las estructuras por edad de la población mundial esperadas para los años 2000 y 2025.

\section{Distribución PORCENTUAL de LA POBLACIÓN MUNDIAL basada EN EL PROMEDIO DE LAS POBLACIONES 2000-2025}

\begin{tabular}{|c|c|}
\hline Grupo de edad & Proporción 2000-2025 \\
\hline $0-4$ & 8.86 \\
\hline $5-9$ & 8.69 \\
\hline $10-14$ & 8.60 \\
\hline $15-19$ & 8.47 \\
\hline $20-24$ & 8.22 \\
\hline $25-29$ & 7.93 \\
\hline $30-34$ & 7.61 \\
\hline $35-39$ & 7.15 \\
\hline $40-44$ & 6.59 \\
\hline $45-49$ & 6.04 \\
\hline $50-54$ & 5.37 \\
\hline $55-59$ & 4.55 \\
\hline $60-64$ & 3.72 \\
\hline $65-69$ & 2.96 \\
\hline $70-74$ & 2.21 \\
\hline $75-79$ & 1.52 \\
\hline $80-84$ & 0.91 \\
\hline $85-89$ & 0.44 \\
\hline $90-94$ & 0.15 \\
\hline $95-99$ & 0.04 \\
\hline $100+$ & 0.005 \\
\hline Total & 100.00 \\
\hline
\end{tabular}

Fuente:W orld Health O rganization. Age standarization of rates: A new W HO standard. GPE Discussion Paper Series N 0.31. Ginebra:W HO, 2000 


\section{Cuadro I \\ Mortalidad GENERAL (CRUdA Y ESTANDARIZADA POR EDAD) POR SEXO, SEGÚN ENTIDAD FEDERATIVA DE RESIDENCIA HABITUAL. MÉXICO, 2001}

\begin{tabular}{|c|c|c|c|c|c|c|c|c|c|c|c|}
\hline & \multicolumn{3}{|c|}{ Total } & \multicolumn{3}{|c|}{ Mujeres } & \multicolumn{3}{|c|}{ Hombres } & \multirow[b]{2}{*}{$\mathrm{NE}$} & \multirow[b]{2}{*}{ ISM } \\
\hline Entidad federativa & Defunciones & Tasa* & Estandarizada ${ }^{\ddagger}$ & Defunciones & Tasa* & Estandarizada ${ }^{\ddagger}$ & Defunciones & Tasa* & Estandarizada ${ }^{\ddagger}$ & & \\
\hline Estados Unidos Mexicanos & 441004 & 4.4 & 6.2 & 196058 & 3.8 & 5.2 & 244606 & 4.9 & 7.1 & 340 & 124.8 \\
\hline Aguascalientes & 3826 & 3.8 & 5.9 & 1799 & 3.5 & 5.3 & 2025 & 4.0 & 6.6 & 2 & 112.6 \\
\hline Baja California & 11090 & 4.5 & 6.9 & 4303 & 3.5 & 5.5 & 6781 & 5.5 & 8.4 & 6 & 157.6 \\
\hline Baja California Sur & 1782 & 4.3 & 6.6 & 684 & 3.4 & 5.1 & 1096 & 5.2 & 8.1 & 2 & 160.2 \\
\hline Campeche & 2493 & 3.4 & 5.4 & 1031 & 2.9 & 4.5 & 1462 & 4.0 & 6.2 & 0 & 141.8 \\
\hline Coahuila & 10748 & 4.5 & 6.4 & 4897 & 4.1 & 5.7 & 5848 & 4.8 & 7.2 & 3 & 119.4 \\
\hline Colima & 2449 & 4.4 & 5.9 & 1083 & 3.9 & 5.1 & 1364 & 4.9 & 6.8 & 2 & 125.9 \\
\hline Chiapas & 15815 & 3.8 & 6.5 & 6942 & 3.4 & 5.8 & 8842 & 4.3 & 7.3 & 31 & 127.4 \\
\hline Chihuahua & 15905 & 5.1 & 7.1 & 6693 & 4.3 & 5.9 & 9172 & 5.9 & 8.3 & 40 & 137.0 \\
\hline Distrito Federal & 46627 & 5.3 & 6.4 & 22728 & 5.0 & 5.6 & 23886 & 5.6 & 7.3 & 13 & 105.1 \\
\hline Durango & 5902 & 3.8 & 5.5 & 2533 & 3.2 & 4.5 & 3349 & 4.4 & 6.5 & 20 & 132.2 \\
\hline Guanajuato & 20375 & 4.1 & 5.8 & 9380 & 3.7 & 5.0 & 10988 & 4.5 & 6.6 & 7 & 117.1 \\
\hline Guerrero & 10715 & 3.3 & 4.9 & 4607 & 2.8 & 4.1 & 6102 & 3.8 & 5.9 & 6 & 132.5 \\
\hline Hidalgo & 9693 & 4.1 & 5.7 & 4268 & 3.6 & 4.8 & 5420 & 4.6 & 6.7 & 5 & 127.0 \\
\hline Jalisco & 30741 & 4.7 & 6.1 & 13880 & 4.1 & 5.2 & 16843 & 5.2 & 7.2 & 18 & 121.3 \\
\hline México & 53315 & 4.0 & 6.4 & 23801 & 3.5 & 5.6 & 29473 & 4.4 & 7.4 & 41 & 123.8 \\
\hline Michoacán & 18277 & 4.3 & 5.7 & 8060 & 3.7 & 4.8 & 10208 & 4.8 & 6.7 & 9 & 126.7 \\
\hline Morelos & 6795 & 4.2 & 5.7 & 3106 & 3.8 & 4.9 & 3678 & 4.6 & 6.5 & 11 & 118.4 \\
\hline $\mathrm{N}$ ayarit & 4136 & 4.3 & 5.4 & 1775 & 3.7 & 4.5 & 2359 & 4.9 & 6.3 & 2 & 132.9 \\
\hline Nuevo León & 16286 & 4.1 & 5.9 & 7277 & 3.7 & 5.0 & 9003 & 4.6 & 6.9 & 6 & 123.7 \\
\hline 0 axaca & 17103 & 4.7 & 6.2 & 7637 & 4.2 & 5.3 & 9435 & 5.3 & 7.2 & 31 & 123.5 \\
\hline Puebla & 25766 & 4.9 & 6.7 & 11534 & 4.3 & 5.6 & 14220 & 5.5 & 8.0 & 12 & 123.3 \\
\hline Q uerétaro & 6022 & 4.2 & 6.5 & 2581 & 3.5 & 5.3 & 3439 & 4.8 & 7.8 & 2 & 133.2 \\
\hline Q uintana Roo & 2493 & 3.0 & 6.2 & 906 & 2.2 & 4.9 & 1574 & 3.7 & 7.4 & 13 & 173.7 \\
\hline San Luis Potosí & 9965 & 4.1 & 5.5 & 4438 & 3.6 & 4.7 & 5522 & 4.5 & 6.3 & 5 & 124.4 \\
\hline Sinaloa & 10034 & 4.0 & 5.4 & 4056 & 3.2 & 4.3 & 5974 & 4.7 & 6.5 & 4 & 147.3 \\
\hline Sonora & 10812 & 4.7 & 6.6 & 4423 & 3.9 & 5.4 & 6382 & 5.5 & 7.8 & 7 & 144.3 \\
\hline Tabasco & 7636 & 3.9 & 6.7 & 3221 & 3.3 & 5.7 & 4406 & 4.5 & 7.7 & 9 & 136.8 \\
\hline Tamaulipas & 11796 & 4.2 & 5.9 & 5135 & 3.7 & 5.0 & 6657 & 4.8 & 6.9 & 4 & 129.6 \\
\hline Tlaxcala & 4487 & 4.5 & 6.1 & 2026 & 4.0 & 5.3 & 2459 & 4.9 & 6.9 & 2 & 121.4 \\
\hline Veracruz & 33203 & 4.6 & 6.2 & 14571 & 4.0 & 5.2 & 18627 & 5.2 & 7.3 & 5 & 127.8 \\
\hline Yucatán & 8357 & 4.9 & 6.1 & 3855 & 4.5 & 5.4 & 4499 & 5.3 & 6.9 & 3 & 116.7 \\
\hline Zacatecas & 6360 & 4.3 & 5.5 & 2828 & 3.8 & 4.7 & 3513 & 4.9 & 6.5 & 19 & 124.2 \\
\hline
\end{tabular}

* Tasa por 1000 habitantes según sexo

₹ Tasa por 1000 habitantes estandarizada por el método directo usando la población mundial estándar W ord Health O rganization.Age Standardization of rates:A new W HO standard

Los totales no incluyen 2123 defunciones de residentes en el extranjero

$N E=$ no especificado

ISM = Indice de sobremortalidad masculina

Fuente: Instituto Nacional de Estadística, Geografía e Informática/D irección General de Información y Evaluación del Desempeño. Secretaría de Salud, México 


\section{Cuadro II \\ Distribución de LAS DEFUnCIONES DE MUJERES POR GRUPOS DE EDAD, SEGÚN ENTIDAD FEDERATIVA De Residencia habitual. MÉxico, 2001}

\begin{tabular}{|c|c|c|c|c|c|c|c|c|c|c|c|}
\hline Entidad federativa & Total & $0-4$ & $5-14$ & $15-24$ & $25-34$ & $35-44$ & $45-54$ & $55-64$ & $65-74$ & $75 y+$ & $\mathrm{Ne}$ \\
\hline Estados Unidos Mexicanos & 196058 & 9.4 & 1.5 & 2.5 & 3.1 & 4.8 & 7.8 & 12.7 & 18.2 & 39.7 & 0.3 \\
\hline Aguascalientes & 1799 & 9.8 & 1.3 & 1.7 & 2.6 & 4.6 & 7.9 & 13.3 & 15.8 & 42.5 & 0.5 \\
\hline Baja C alifornia & 4303 & 11.6 & 1.4 & 2.8 & 4.0 & 6.5 & 9.1 & 13.9 & 17.3 & 33.2 & 0.2 \\
\hline Baja California Sur & 684 & 11.0 & 1.5 & 2.0 & 3.5 & 5.6 & 8.9 & 12.3 & 17.4 & 37.4 & 0.4 \\
\hline Campeche & 1031 & 9.3 & 1.8 & 2.5 & 3.0 & 5.6 & 9.7 & 13.9 & 15.5 & 37.2 & 1.4 \\
\hline Coahuila & 4897 & 6.1 & 1.0 & 2.1 & 2.8 & 4.7 & 8.7 & 14.9 & 20.0 & 39.3 & 0.4 \\
\hline Colima & 1083 & 7.8 & 1.3 & 2.5 & 2.3 & 4.7 & 8.7 & 10.6 & 17.4 & 44.2 & 0.5 \\
\hline Chiapas & 6942 & 13.2 & 3.0 & 4.5 & 5.0 & 6.8 & 8.8 & 13.1 & 15.9 & 29.4 & 0.3 \\
\hline Chihuahua & 6693 & 9.7 & 1.2 & 2.4 & 3.6 & 5.2 & 7.7 & 13.7 & 20.1 & 36.1 & 0.5 \\
\hline Distrito Federal & 22728 & 6.3 & 0.8 & 2.0 & 2.5 & 4.1 & 7.4 & 12.7 & 19.8 & 44.3 & 0.0 \\
\hline Durango & 2533 & 3.0 & 1.1 & 2.5 & 2.6 & 4.3 & 7.9 & 14.7 & 23.3 & 40.2 & 0.4 \\
\hline Guanajuato & 9380 & 11.1 & 1.3 & 2.3 & 2.5 & 4.3 & 6.5 & 11.8 & 16.5 & 42.8 & 0.7 \\
\hline Guerrero & 4607 & 6.5 & 1.4 & 3.3 & 4.7 & 6.1 & 9.3 & 13.7 & 19.6 & 35.4 & 0.2 \\
\hline Hidalgo & 4268 & 8.9 & 1.5 & 2.9 & 3.3 & 5.5 & 8.3 & 12.3 & 17.2 & 39.5 & 0.7 \\
\hline Jalisco & 13880 & 8.2 & 1.4 & 2.0 & 2.4 & 4.1 & 7.3 & 11.7 & 16.9 & 45.7 & 0.2 \\
\hline México & 23801 & 13.9 & 1.6 & 2.9 & 3.8 & 5.8 & 8.7 & 13.5 & 17.2 & 32.5 & 0.1 \\
\hline Michoacán & 8060 & 7.4 & 1.7 & 2.4 & 2.9 & 4.6 & 6.7 & 11.0 & 18.5 & 44.4 & 0.4 \\
\hline Morelos & 3106 & 7.0 & 1.4 & 2.6 & 2.9 & 4.6 & 6.8 & 13.3 & 18.6 & 42.3 & 0.5 \\
\hline $\mathrm{N}$ ayarit & 1775 & 6.5 & 1.1 & 1.9 & 3.1 & 4.3 & 6.9 & 12.1 & 15.8 & 48.1 & 0.3 \\
\hline N uevo León & 7277 & 7.1 & 1.0 & 1.6 & 2.4 & 4.3 & 7.4 & 13.4 & 19.5 & 42.8 & 0.5 \\
\hline 0 axaca & 7637 & 9.3 & 2.0 & 2.8 & 3.4 & 4.9 & 7.5 & 12.0 & 18.1 & 39.8 & 0.3 \\
\hline Puebla & 11534 & 14.5 & 1.7 & 2.4 & 3.3 & 4.7 & 7.2 & 10.9 & 16.8 & 38.1 & 0.2 \\
\hline Q uerétaro & 2581 & 13.2 & 1.9 & 3.4 & 3.5 & 5.3 & 7.2 & 12.1 & 15.1 & 37.6 & 0.7 \\
\hline Q uintana Roo & 906 & 16.8 & 2.1 & 5.1 & 5.8 & 7.7 & 10.5 & 14.2 & 14.7 & 22.7 & 0.3 \\
\hline San Luis Potosí & 4438 & 9.4 & 1.4 & 2.3 & 2.9 & 4.2 & 6.6 & 11.5 & 17.8 & 43.6 & 0.3 \\
\hline Sinaloa & 4056 & 4.3 & 1.2 & 2.3 & 2.4 & 4.1 & 8.2 & 13.1 & 18.0 & 45.9 & 0.5 \\
\hline Sonora & 4423 & 8.6 & 1.3 & 2.5 & 2.5 & 3.9 & 8.1 & 14.7 & 19.4 & 39.0 & 0.1 \\
\hline Tabasco & 3221 & 12.2 & 1.9 & 3.3 & 3.8 & 5.2 & 8.6 & 13.3 & 17.4 & 33.9 & 0.3 \\
\hline Tamaulipas & 5135 & 6.0 & 1.5 & 2.1 & 3.3 & 5.1 & 7.9 & 14.3 & 20.4 & 38.8 & 0.5 \\
\hline Tlaxcala & 2026 & 13.2 & 1.6 & 2.3 & 2.5 & 4.6 & 6.6 & 11.2 & 16.8 & 41.0 & 0.2 \\
\hline Veracruz & 14571 & 8.0 & 1.6 & 2.5 & 3.4 & 4.7 & 8.2 & 13.7 & 19.5 & 38.0 & 0.5 \\
\hline Yucatán & 3855 & 8.2 & 1.1 & 1.7 & 1.7 & 3.3 & 7.0 & 11.3 & 17.1 & 48.0 & 0.8 \\
\hline Zacatecas & 2828 & 8.4 & 1.4 & 2.1 & 2.7 & 3.8 & 6.9 & 9.4 & 17.7 & 47.2 & 0.4 \\
\hline
\end{tabular}

Los totales no incluyen 731 defunciones de mujeres residentes en el extranjero $\mathrm{NE}=$ no especificado

Fuente: Instituto Nacional de Estadística, Geografía e Informática/Dirección General de Información y Evaluación del Desempeño. Secretaría de Salud, México 


\section{Cuadro III \\ Distribución de LAS Defunciones de hombres PoR GRUPOS De EDAD, SEgún ENTIDAD FEDERATIVA De Residencia habitual. MéXico, 2001}

\begin{tabular}{|c|c|c|c|c|c|c|c|c|c|c|c|}
\hline Entidad federativa & Total & $0-4$ & $5-14$ & $15-24$ & $25-34$ & $35-44$ & $45-54$ & $55-64$ & $65-74$ & $75 y+$ & $\mathrm{Ne}$ \\
\hline Estados Unidos Mexicanos & 244606 & 9.8 & 1.7 & 5.0 & 6.8 & 8.0 & 10.1 & 13.1 & 16.8 & 28.1 & 0.6 \\
\hline Aguascalientes & 2025 & 11.0 & 1.8 & 4.8 & 5.7 & 7.0 & 8.7 & 12.7 & 15.2 & 32.3 & 0.8 \\
\hline Baja California & 6781 & 10.7 & 1.4 & 4.9 & 10.7 & 11.7 & 11.9 & 13.0 & 14.8 & 19.8 & 1.2 \\
\hline Baja California Sur & 1096 & 11.9 & 2.3 & 5.6 & 6.7 & 7.0 & 10.0 & 13.3 & 16.0 & 26.5 & 0.8 \\
\hline Campeche & 1462 & 7.5 & 1.7 & 5.3 & 6.8 & 7.0 & 12.0 & 11.9 & 16.3 & 29.7 & 1.7 \\
\hline Coahuila & 5848 & 6.5 & 1.4 & 4.3 & 5.6 & 6.8 & 9.6 & 15.8 & 20.3 & 29.2 & 0.5 \\
\hline Colima & 1364 & 7.4 & 1.6 & 5.0 & 6.5 & 7.1 & 8.8 & 12.6 & 18.3 & 31.6 & 1.0 \\
\hline Chiapas & 8842 & 12.9 & 2.8 & 6.9 & 8.7 & 9.0 & 9.9 & 11.7 & 13.8 & 23.0 & 1.3 \\
\hline Chihuahua & 9172 & 9.2 & 1.5 & 5.8 & 8.6 & 8.7 & 10.0 & 13.7 & 17.2 & 24.2 & 1.1 \\
\hline Distrito Federal & 23886 & 7.6 & 0.9 & 3.9 & 6.4 & 8.3 & 11.0 & 14.3 & 18.4 & 29.1 & 0.0 \\
\hline Durango & 3349 & 3.1 & 1.4 & 5.9 & 6.7 & 6.7 & 8.8 & 13.7 & 21.0 & 32.4 & 0.3 \\
\hline Guanajuato & 10988 & 12.1 & 2.1 & 5.3 & 5.4 & 6.9 & 8.7 & 10.9 & 16.0 & 31.9 & 0.7 \\
\hline Guerrero & 6102 & 6.1 & 1.8 & 5.5 & 8.8 & 9.2 & 10.8 & 12.9 & 17.2 & 27.1 & 0.6 \\
\hline Hidalgo & 5420 & 9.0 & 2.1 & 4.6 & 5.8 & 8.2 & 11.0 & 14.8 & 15.3 & 28.7 & 0.6 \\
\hline Jalisco & 16843 & 9.0 & 1.9 & 4.5 & 6.4 & 7.1 & 9.2 & 12.5 & 15.8 & 33.0 & 0.6 \\
\hline México & 29473 & 14.9 & 1.6 & 5.5 & 7.2 & 9.0 & 11.4 & 13.6 & 15.5 & 21.1 & 0.1 \\
\hline Michoacán & 10208 & 7.9 & 2.3 & 5.5 & 6.5 & 7.4 & 8.9 & 11.9 & 16.3 & 32.9 & 0.4 \\
\hline Morelos & 3678 & 7.3 & 1.6 & 4.9 & 5.8 & 7.6 & 10.2 & 12.0 & 19.2 & 30.5 & 0.9 \\
\hline $\mathrm{N}$ ayarit & 2359 & 6.1 & 1.9 & 5.3 & 6.4 & 6.9 & 8.2 & 11.1 & 17.6 & 36.1 & 0.4 \\
\hline N uevo León & 9003 & 7.4 & 1.1 & 4.0 & 5.5 & 6.5 & 9.4 & 15.0 & 19.8 & 30.5 & 0.9 \\
\hline 0 axaca & 9435 & 9.5 & 2.6 & 5.1 & 7.1 & 8.1 & 11.0 & 11.8 & 15.9 & 28.3 & 0.6 \\
\hline Puebla & 14220 & 15.6 & 2.0 & 4.3 & 5.8 & 8.0 & 10.3 & 12.6 & 14.5 & 26.6 & 0.3 \\
\hline Querétaro & 3439 & 11.4 & 2.4 & 6.0 & 7.7 & 8.9 & 11.1 & 12.9 & 13.8 & 24.9 & 0.7 \\
\hline Q uintana Roo & 1574 & 14.4 & 2.0 & 6.9 & 10.4 & 11.4 & 11.1 & 10.1 & 11.8 & 17.7 & 4.3 \\
\hline San Luis Potosí & 5522 & 9.7 & 1.8 & 5.1 & 5.3 & 6.5 & 8.9 & 11.0 & 17.1 & 34.1 & 0.5 \\
\hline Sinaloa & 5974 & 3.2 & 1.6 & 5.9 & 8.0 & 7.4 & 9.0 & 13.7 & 19.6 & 31.0 & 0.5 \\
\hline Sonora & 6382 & 7.8 & 1.2 & 4.4 & 6.6 & 7.8 & 9.5 & 14.4 & 19.7 & 28.3 & 0.3 \\
\hline Tabasco & 4406 & 11.7 & 2.1 & 7.1 & 8.1 & 8.8 & 9.4 & 11.3 & 15.4 & 25.2 & 0.8 \\
\hline Tamaulipas & 6657 & 6.0 & 1.4 & 4.6 & 6.0 & 7.0 & 9.9 & 15.0 & 21.0 & 28.2 & 1.0 \\
\hline Tlaxcala & 2459 & 15.7 & 1.9 & 5.3 & 5.3 & 7.2 & 8.8 & 11.1 & 13.9 & 30.0 & 0.7 \\
\hline $\begin{array}{l}\text { Veracruz } \\
\text {. }\end{array}$ & 18627 & 7.7 & 1.7 & 4.1 & 6.4 & 8.8 & 11.1 & 14.3 & 17.8 & 27.4 & 0.8 \\
\hline Yucatán & 4499 & 8.0 & 1.5 & 2.8 & 5.2 & 6.2 & 8.2 & 11.3 & 16.8 & 39.1 & 0.9 \\
\hline Zacatecas & 3513 & 8.3 & 2.0 & 5.8 & 5.6 & 5.1 & 7.6 & 10.1 & 16.8 & 38.2 & 0.5 \\
\hline
\end{tabular}

Los totales no incluyen 1392 defunciones de hombres residentes en el extranjero

$\mathrm{NE}=$ no especificado

Fuente: Instituto Nacional de Estadística, Geografía e Informática/Dirección General de Información y Evaluación del Desempeño. Secretaría de Salud, México 


\section{Cuadro IV \\ TASA DE MORTALIDAD (ESTANDARIZADA POR EDAD) POR GRANDES GRUPOS DE CAUSAS, SEgÚN SEXO Y ENTIDAD Federativa. MÉXICO, 2001}

\begin{tabular}{|c|c|c|c|c|c|c|c|c|c|}
\hline \multirow[b]{2}{*}{ Entidad federativa } & \multicolumn{3}{|c|}{$\begin{array}{l}\text { Transmisibles, nutricionales } \\
\text { y de la reproducción* }\end{array}$} & \multicolumn{3}{|c|}{ No transmisibles ${ }^{\ddagger}$} & \multicolumn{3}{|c|}{ Lesiones ${ }^{\S}$} \\
\hline & Total & M ujeres & Hombres & Total & Mujeres & Hombres & Total & Mujeres & Hombres \\
\hline Estados Unidos Mexicanos & 73.8 & 64.4 & 83.6 & 471.4 & 423.8 & 523.5 & 57.5 & 23.3 & 93.5 \\
\hline A guascalientes & 59.0 & 54.0 & 64.3 & 474.7 & 445.1 & 508.4 & 48.7 & 22.5 & 76.5 \\
\hline Baja California & 78.4 & 57.5 & 99.2 & 522.1 & 450.4 & 596.0 & 77.5 & 31.2 & 124.3 \\
\hline Baja California Sur & 80.0 & 65.6 & 93.1 & 517.6 & 423.0 & 614.4 & 59.0 & 18.9 & 97.5 \\
\hline Campeche & 57.9 & 48.5 & 67.5 & 407.0 & 362.6 & 451.7 & 52.2 & 22.0 & 81.9 \\
\hline Coahuila & 58.8 & 55.8 & 61.2 & 523.7 & 480.8 & 569.2 & 49.0 & 22.0 & 76.1 \\
\hline Colima & 63.2 & 53.7 & 72.8 & 462.5 & 417.0 & 509.0 & 59.3 & 26.1 & 93.8 \\
\hline Chiapas & 115.8 & 107.3 & 124.0 & 421.6 & 403.6 & 440.2 & 62.6 & 20.1 & 105.2 \\
\hline Chihuahua & 78.8 & 66.5 & 91.2 & 538.0 & 483.7 & 592.5 & 79.6 & 29.4 & 131.0 \\
\hline Distrito Federal & 70.9 & 60.0 & 82.5 & 515.0 & 471.9 & 565.2 & 45.8 & 21.6 & 72.7 \\
\hline Durango & 43.4 & 34.2 & 53.3 & 430.6 & 384.2 & 480.2 & 54.7 & 18.7 & 92.2 \\
\hline Guanajuato & 69.2 & 61.9 & 77.1 & 447.5 & 406.0 & 495.0 & 51.6 & 23.3 & 82.6 \\
\hline Guerrero & 64.1 & 58.1 & 70.5 & 339.6 & 307.6 & 375.4 & 72.3 & 26.1 & 122.2 \\
\hline Hidalgo & 61.2 & 58.1 & 64.0 & 445.4 & 388.5 & 506.3 & 57.3 & 24.7 & 91.0 \\
\hline Jalisco & 68.6 & 59.0 & 78.7 & 481.9 & 431.8 & 538.0 & 59.1 & 24.8 & 96.5 \\
\hline México & 84.4 & 74.6 & 94.7 & 501.5 & 453.1 & 555.1 & 54.5 & 24.5 & 86.5 \\
\hline Michoacán & 56.8 & 51.2 & 62.5 & 429.5 & 388.7 & 474.5 & 71.7 & 27.4 & 119.3 \\
\hline Morelos & 67.9 & 60.6 & 75.6 & 436.2 & 397.7 & 478.7 & 57.2 & 26.3 & 90.4 \\
\hline $\bar{N}$ ayarit & 62.1 & 52.1 & 72.7 & 403.4 & 365.3 & 444.0 & 63.5 & 22.2 & 105.5 \\
\hline N uevo León & 55.6 & 47.4 & 64.0 & 487.0 & 432.2 & 547.0 & 43.2 & 19.4 & 67.7 \\
\hline 0 axaca & 103.4 & 95.5 & 111.3 & 417.4 & 377.8 & 460.0 & 69.4 & 23.3 & 118.7 \\
\hline Puebla & 98.7 & 85.9 & 112.7 & 494.4 & 428.5 & 569.7 & 55.3 & 23.1 & 90.8 \\
\hline Q uerétaro & 73.2 & 65.7 & 80.2 & 493.0 & 423.4 & 569.7 & 67.4 & 25.6 & 112.0 \\
\hline Q uintana Roo & 82.5 & 63.7 & 100.9 & 440.4 & 385.3 & 491.2 & 76.4 & 28.0 & 121.2 \\
\hline San Luis Potosí & 65.4 & 58.4 & 72.9 & 414.9 & 377.1 & 455.8 & 53.3 & 18.8 & 88.9 \\
\hline Sinaloa & 44.2 & 38.0 & 50.4 & 423.0 & 362.0 & 485.8 & 65.7 & 22.1 & 109.0 \\
\hline Sonora & 70.7 & 60.8 & 80.3 & 519.3 & 450.5 & 588.7 & 63.6 & 23.0 & 103.5 \\
\hline Tabasco & 73.4 & 64.1 & 82.3 & 506.5 & 472.3 & 542.5 & 75.9 & 25.0 & 128.1 \\
\hline Tamaulipas & 55.0 & 44.3 & 66.2 & 474.2 & 421.7 & 531.3 & 51.7 & 21.6 & 82.7 \\
\hline Tlaxcala & 89.8 & 79.7 & 100.7 & 447.8 & 412.4 & 485.8 & 55.3 & 21.6 & 90.7 \\
\hline Veracruz & 77.2 & 65.3 & 89.9 & 479.4 & 422.0 & 542.2 & 51.0 & 20.2 & 83.5 \\
\hline Yucatán & 81.8 & 67.9 & 96.2 & 458.6 & 424.3 & 495.9 & 44.8 & 21.3 & 69.1 \\
\hline Zacatecas & 55.4 & 54.1 & 56.3 & 426.0 & 379.6 & 475.5 & 57.2 & 21.1 & 95.7 \\
\hline
\end{tabular}

Tasa por 100000 habitantes estandarizada por el método directo usando la población mundial estándar (ver nota metodológica)

* Incluye enfermedades infecciosas y parasitarias, muertes maternas, perinatales y deficiencias nutricionales

A00-B99,G 00-G 04,N 70-N 73,J00-J06,J10-J18,J20-J22,H65-H66,0 00-0 99,P00-P96,E00-E02,E40-E46,E50,D 50-D 64 (CIE 10a revisión)

₹ C 00-D 48,D 65-D 89,E03-E34,E51-F99, G 06-H61,H68-199,J30-N 64,N 75-M 99,Q 00-Q 99 Clasificación Internacional de Enfermedades (CIE) 10a revisión

§ V01-Y89 (CIE 10a revisión)

Los totales no incluyen defunciones de residentes en el extranjero

Fuente: Instituto N acional de Estadística, Geografía e Informática/D irección General de Información y Evaluación del Desempeño. Secretaría de Salud, México 


\section{Cuadro V}

Principales causas de mortalidad en Mujeres. México, 2001

\begin{tabular}{|c|c|c|c|c|c|}
\hline $\begin{array}{l}\text { Número } \\
\text { de orden }\end{array}$ & Causa & $\begin{array}{c}\text { Clave CIE } \\
\text { 10a Revisión }\end{array}$ & Defunciones & Tasa* & $\%$ \\
\hline & Total & A00-Y98 & 196058 & 384.9 & 100.0 \\
\hline 1 & Diabetes mellitus & E10-E14 & 27528 & 54.0 & 14.0 \\
\hline 2 & Enfermedades isquémicas del corazón & $120-125$ & 20391 & 40.0 & 10.4 \\
\hline 3 & Enfermedad cerebrovascular & $160-169$ & 13691 & 26.9 & 7.0 \\
\hline 4 & Ciertas afecciones originadas en el período perinatal & P00-P96 & 7690 & 15.1 & 3.9 \\
\hline 5 & Enfermedad pulmonar obstructiva crónica & J40-J44, J67 & 6861 & 13.5 & 3.5 \\
\hline 6 & Cirrosis y otras enfermedades crónicas del hígado & $\mathrm{K} 70, \mathrm{~K} 72.1, \mathrm{~K} 73, \mathrm{~K} 74, \mathrm{~K} 76$ & 6042 & 11.9 & 3.1 \\
\hline 7 & Infecciones respiratorias agudas bajas & J10-J18, J20-J22 & 6011 & 11.8 & 3.1 \\
\hline 8 & Enfermedades hipertensivas & $|10-| 15$ & 5952 & 11.7 & 3.0 \\
\hline 9 & $\mathrm{~N}$ efritis y nefrosis & N 00-N 19 & 5064 & 9.9 & 2.6 \\
\hline 10 & Tumor maligno del cuello del útero & C53 & 4501 & 8.8 & 2.3 \\
\hline 11 & Desnutrición calórico protéica & E40-E46 & 4399 & 8.6 & 2.2 \\
\hline 12 & Tumor maligno de la mama & C50 & 3574 & 7.0 & 1.8 \\
\hline 13 & Accidentes de tráfico de vehículos de motor & 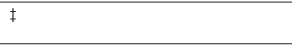 & 3053 & 6.0 & 1.6 \\
\hline 14 & Enfermedades infecciosas intestinales & A00-A09 & 2414 & 4.7 & 1.2 \\
\hline 15 & Tumor maligno del estómago & C16 & 2328 & 4.6 & 1.2 \\
\hline 16 & Tumor maligno del hígado & $\mathrm{C} 22$ & 2301 & 4.5 & 1.2 \\
\hline 17 & Tumor maligno de tráquea, bronquios y pulmón & C 33-C 34 & 2001 & 3.9 & 1.0 \\
\hline 18 & Anemia & D50-D 64 & 1793 & 3.5 & 0.9 \\
\hline 19 & Malformaciones congénitas del corazón & Q 20-Q 24 & 1608 & 3.2 & 0.8 \\
\hline 20 & Leucemia & C91-C95 & 1511 & 3.0 & 0.8 \\
\hline & Causas mal definidas & R00-R99 & 4576 & 9.0 & 2.3 \\
\hline & Las demás & & 62769 & 123.2 & 32.0 \\
\hline
\end{tabular}

* Tasa por 100000 mujeres

₹ V02-V04 (.1, .9),V09.2-V09.3,V09.9,V12-V14 (.3-.9),V19.4-V19.6,V20-V28 (.3-.9),V29-V79 (.4-.9),V80.3-V80.5,V81.1,V82.1,V83-V86 (.0-.3),V87.0-V87.8, V89.2,V89.9, Y85.0. Clasificación Internacional de Enfermedades (CIE) 10a revisión

Los totales no incluyen defunciones de mujeres residentes en el extranjero

Fuente: Instituto Nacional de Estadística, Geografía e Informática/D irección General de Información y Evaluación del Desempeño. Secretaría de Salud, México 


\section{Cuadro VI \\ Principales causas de mortalidad en Hombres. México, 2001}

\begin{tabular}{|c|c|c|c|c|c|}
\hline $\begin{array}{l}\text { Número } \\
\text { de orden }\end{array}$ & Causa & $\begin{array}{l}\text { Clave CIE } \\
\text { 10a Revisión }\end{array}$ & Defunciones & Tasa* & $\%$ \\
\hline & Total & A $00-Y 98$ & 244606 & 488.7 & 100.0 \\
\hline 1 & Enfermedades isquémicas del corazón & $120-125$ & 25011 & 50.0 & 10.2 \\
\hline 2 & Diabetes mellitus & E10-E14 & 22305 & 44.6 & 9.1 \\
\hline 3 & Cirrosis y otras enfermedades crónicas del hígado & $\mathrm{K} 70, \mathrm{~K} 72.1, \mathrm{~K} 73, \mathrm{~K} 74, \mathrm{~K} 76$ & 19649 & 39.3 & 8.0 \\
\hline 4 & Enfermedad cerebrovascular & $160-169$ & 11956 & 23.9 & 4.9 \\
\hline 5 & Accidentes de tráfico de vehículos de motor & $1 /$ & 10697 & 21.4 & 4.4 \\
\hline 6 & Ciertas afecciones originadas en el período perinatal & P00-P96 & 10452 & 20.9 & 4.3 \\
\hline 7 & Enfermedad pulmonar obstructiva crónica & J40-J44, J67 & 9079 & 18.1 & 3.7 \\
\hline 8 & Agresiones (homicidios) & $X 85-Y 09, Y 87.1$ & 8885 & 17.8 & 3.6 \\
\hline 9 & Infecciones respiratorias agudas bajas & J10-J18, J20-J22 & 7079 & 14.1 & 2.9 \\
\hline 10 & $N$ efritis y nefrosis & N 00-N 19 & 5407 & 10.8 & 2.2 \\
\hline 11 & Tumor maligno de tráquea, bronquios y pulmón & C 33-C 34 & 4400 & 8.8 & 1.8 \\
\hline 12 & Enfermedades hipertensivas & $|10-| 15$ & 4218 & 8.4 & 1.7 \\
\hline 13 & Desnutrición calórico protéica & E40-E46 & 4214 & 8.4 & 1.7 \\
\hline 14 & Tumor maligno de la próstata & C61 & 4015 & 8.0 & 1.6 \\
\hline 15 & Uso de alcohol & F10, G31.2 & 3955 & 7.9 & 1.6 \\
\hline 16 & $\mathrm{VIH} / \mathrm{SIDA}$ & B20-B24 & 3615 & 7.2 & 1.5 \\
\hline 17 & Lesiones auto infligidas intencionalmente (suicidios) & $X 60-X 84, Y 87.0$ & 3110 & 6.2 & 1.3 \\
\hline 18 & Tumor maligno del estómago & $\mathrm{C} 16$ & 2656 & 5.3 & 1.1 \\
\hline 19 & Enfermedades infecciosas intestinales & A00-A09 & 2479 & 5.0 & 1.0 \\
\hline 20 & Tuberculosis & A15-A19, B90 & 2284 & 4.6 & 0.9 \\
\hline & Causas mal definidas & R00-R99 & 4597 & 9.2 & 1.9 \\
\hline & Las demás & & 74543 & 148.9 & 30.5 \\
\hline
\end{tabular}

* Tasa por 100000 habitantes

₹ V02-V04 (.1, .9),V09.2-V09.3,V09.9,V12-V14 (.3-.9),V19.4-V19.6,V20-V28 (.3-.9),V29-V79 (.4-.9),V80.3-V80.5,V81.1,V82.1,V83-V86 (.0-.3),V87.0-V87.8, V89.2,V89.9, Y85.0. Clasificación Internacional de Enfermedades (CIE) 10a revisión

Los totales no incluyen defunciones de hombres residentes en el extranjero

Fuente: Instituto Nacional de Estadística, Geografía e Informática/Dirección General de Información y Evaluación del Desempeño. Secretaría de Salud, México 


\section{Cuadro VII \\ MoRTALIDAD EN MUJERES (ESTANDARIZADA POR EDAD) POR ENFERMEDADES TRANSMISIBLES, PERINATALES Y DEFICIENCIAS DE LA NUTRICIÓN, SEGÚN ENTIDAD FEDERATIVA DE RESIDENCIA HABITUAL , 2001}

\begin{tabular}{|c|c|c|c|c|c|c|c|c|c|c|c|c|c|c|}
\hline Entidad federativa & $\begin{array}{r}\text { Enferme } \\
\text { infeccio } \\
\text { intestin } \\
\text { A00-A } \\
\text { Defuciones }\end{array}$ & $\begin{array}{l}\text { dades } \\
\text { osas } \\
\text { ales } \\
09^{\ddagger} \\
\text { Tasa }\end{array}$ & $\begin{array}{r}\text { Infeccic } \\
\text { respirat } \\
\text { aguda } \\
\text { J00-J2 } \\
\text { Defuciones }\end{array}$ & $\begin{array}{l}\text { ones } \\
\text { orias } \\
\text { as* } \\
2^{\ddagger} \\
\text { Tasa }\end{array}$ & $\begin{array}{r}\text { Tuberc } \\
\text { pulmo } \\
\text { A15-A } \\
\text { Defuciones }\end{array}$ & $\begin{array}{l}\text { ulosis } \\
\text { onar } \\
116^{\ddagger} \\
\text { Tasa }\end{array}$ & $\begin{array}{r}\text { SID } \\
\text { B20-B } \\
\text { Defuciones }\end{array}$ & & $\begin{array}{r}\text { Ciert } \\
\text { afeccio } \\
\text { perinat } \\
\text { P00-p96 } \\
\text { Defuciones }\end{array}$ & $\begin{array}{l}\text { as } \\
\text { nes } \\
\text { ales } \\
\text { A33 } \\
\text { Tasa }\end{array}$ & $\begin{array}{r}\text { Deficier } \\
\text { de } \\
\text { nutric } \\
\text { E40-E } \\
\text { Defuciones }\end{array}$ & $\begin{array}{l}\text { ncias } \\
\text { la } \\
\text { ión } \\
64^{\ddagger} \\
\text { Tasa }\end{array}$ & $\begin{array}{l}\text { Anem } \\
\text { D50-D } \\
\text { Defuciones }\end{array}$ & $\begin{array}{l}\text { mia } \\
\text { D64 } \\
\text { Tasa }\end{array}$ \\
\hline$N$ acional & 2414 & 5.5 & 6238 & 14.8 & 952 & 2.6 & 701 & 1.5 & 7693 & 13.0 & 4417 & 10.8 & 719 & 1.8 \\
\hline Aguascalientes & 21 & 6.1 & 18 & 4.3 & 4 & 1.2 & 4 & 1.0 & 93 & 14.3 & 49 & 12.9 & 9 & 2.1 \\
\hline Baja California & 29 & 3.0 & 107 & 12.5 & 37 & 4.1 & 37 & 2.9 & 177 & 13.1 & 54 & 7.2 & 4 & 0.5 \\
\hline Baja California Sur & 5 & 2.5 & 31 & 20.5 & 1 & 0.7 & 3 & 1.5 & 26 & 12.1 & 17 & 12.8 & 0 & \#DIV/0! \\
\hline Campeche & 14 & 5.1 & 23 & 10.2 & 5 & 2.3 & 7 & 2.3 & 47 & 11.4 & 18 & 8.0 & 3 & 1.0 \\
\hline Coahuila & 40 & 4.0 & 113 & 11.9 & 20 & 2.3 & 7 & 0.6 & 116 & 8.3 & 95 & 10.5 & 8 & 0.9 \\
\hline Colima & 4 & 1.6 & 28 & 11.4 & 7 & 3.4 & 9 & 3.3 & 31 & 10.3 & 31 & 12.7 & 2 & 0.7 \\
\hline Chiapas & 291 & 18.0 & 348 & 25.1 & 123 & 10.1 & 41 & 2.3 & 340 & 11.9 & 172 & 14.2 & 53 & 4.2 \\
\hline Chihuahua & 54 & 4.2 & 233 & 18.5 & 29 & 2.4 & 21 & 1.3 & 277 & 16.1 & 82 & 7.0 & 18 & 1.6 \\
\hline Distrito Federal & 145 & 3.4 & 764 & 17.4 & 34 & 0.9 & 80 & 1.8 & 635 & 15.5 & 272 & 5.9 & 31 & 0.7 \\
\hline Durango & 30 & 5.4 & 60 & 9.9 & 7 & 1.3 & 3 & 0.4 & 28 & 2.9 & 35 & 5.7 & 4 & 0.7 \\
\hline Guanajuato & 138 & 5.9 & 290 & 13.1 & 16 & 0.9 & 27 & 1.3 & 468 & 14.5 & 299 & 13.6 & 40 & 2.0 \\
\hline Guerrero & 86 & 6.6 & 105 & 8.6 & 46 & 4.1 & 32 & 2.4 & 108 & 5.0 & 151 & 11.9 & 35 & 2.9 \\
\hline Hidalgo & 27 & 2.6 & 110 & 10.9 & 22 & 2.8 & 8 & 0.7 & 172 & 11.9 & 135 & 13.8 & 33 & 3.4 \\
\hline Jalisco & 112 & 3.7 & 463 & 15.1 & 42 & 1.7 & 49 & 1.7 & 462 & 11.8 & 318 & 10.0 & 25 & 0.8 \\
\hline México & 355 & 6.7 & 1020 & 19.9 & 63 & 1.5 & 70 & 1.1 & 1404 & 18.7 & 486 & 11.1 & 59 & 1.4 \\
\hline Michoacán & 82 & 4.0 & 243 & 12.7 & 13 & 0.8 & 14 & 0.7 & 234 & 8.5 & 177 & 9.3 & 39 & 2.2 \\
\hline Morelos & 33 & 4.8 & 71 & 10.1 & 11 & 1.6 & 14 & 1.7 & 107 & 12.5 & 88 & 13.0 & 12 & 1.9 \\
\hline$N$ ayarit & 11 & 2.5 & 54 & 12.3 & 16 & 4.5 & 10 & 2.2 & 30 & 5.4 & 51 & 10.9 & 1 & 0.2 \\
\hline Nuevo León & 41 & 2.7 & 177 & 11.4 & 41 & 2.7 & 16 & 0.9 & 200 & 9.7 & 86 & 5.6 & 3 & 0.2 \\
\hline 0 axaca & 217 & 13.1 & 297 & 19.1 & 75 & 5.6 & 24 & 1.4 & 242 & 10.3 & 364 & 23.7 & 65 & 4.3 \\
\hline Puebla & 172 & 6.7 & 439 & 17.4 & 52 & 2.7 & 33 & 1.4 & 740 & 20.6 & 376 & 16.8 & 77 & 3.7 \\
\hline Q uerétaro & 36 & 6.4 & 58 & 10.0 & 13 & 3.0 & 3 & 0.4 & 134 & 14.8 & 92 & 17.8 & 6 & 1.3 \\
\hline Q uintana Roo & 16 & 5.1 & 21 & 13.4 & 7 & 4.8 & 11 & 2.4 & 77 & 14.5 & 14 & 9.6 & 1 & 1.0 \\
\hline San Luis Potosí & 66 & 6.3 & 130 & 11.6 & 25 & 2.8 & 8 & 0.7 & 146 & 9.0 & 105 & 9.8 & 13 & 1.2 \\
\hline Sinaloa & 38 & 3.9 & 106 & 10.5 & 27 & 2.8 & 6 & 0.5 & 46 & 3.6 & 71 & 6.6 & 6 & 0.7 \\
\hline Sonora & 35 & 3.9 & 101 & 11.6 & 18 & 2.1 & 6 & 0.5 & 166 & 14.0 & 101 & 12.0 & 5 & 0.7 \\
\hline Tabasco & 24 & 2.1 & 74 & 11.8 & 20 & 3.4 & 21 & 2.2 & 180 & 14.3 & 85 & 15.0 & 20 & 3.8 \\
\hline Tamaulipas & 21 & 1.8 & 120 & 11.3 & 24 & 2.4 & 16 & 1.1 & 156 & 10.6 & 56 & 5.2 & 8 & 0.7 \\
\hline Tlaxcala & 24 & 4.8 & 95 & 21.4 & 6 & 1.8 & 6 & 1.2 & 115 & 19.5 & 55 & 12.6 & 4 & 1.0 \\
\hline Veracruz & 152 & 4.8 & 331 & 10.7 & 130 & 4.6 & 95 & 2.8 & 491 & 12.4 & 344 & 11.5 & 110 & 3.8 \\
\hline Yucatán & 54 & 7.2 & 103 & 13.3 & 12 & 1.9 & 16 & 1.9 & 131 & 14.7 & 83 & 10.3 & 14 & 1.9 \\
\hline Zacatecas & 41 & 5.8 & 105 & 15.6 & 6 & 1.0 & 4 & 0.6 & 114 & 12.3 & 55 & 7.8 & 11 & 1.5 \\
\hline \multicolumn{15}{|c|}{$\begin{array}{l}\text { * Incluye neumonía e influenza } \\
\text { ₹ Códigos de la Clasificación Internacional de Enfermedades (CIE) 10a revisión }\end{array}$} \\
\hline
\end{tabular}

Fuente: Instituto Nacional de Estadística, Geografía e Informática/D irección General de Información y Evaluación del Desempeño. Secretaría de Salud, México 

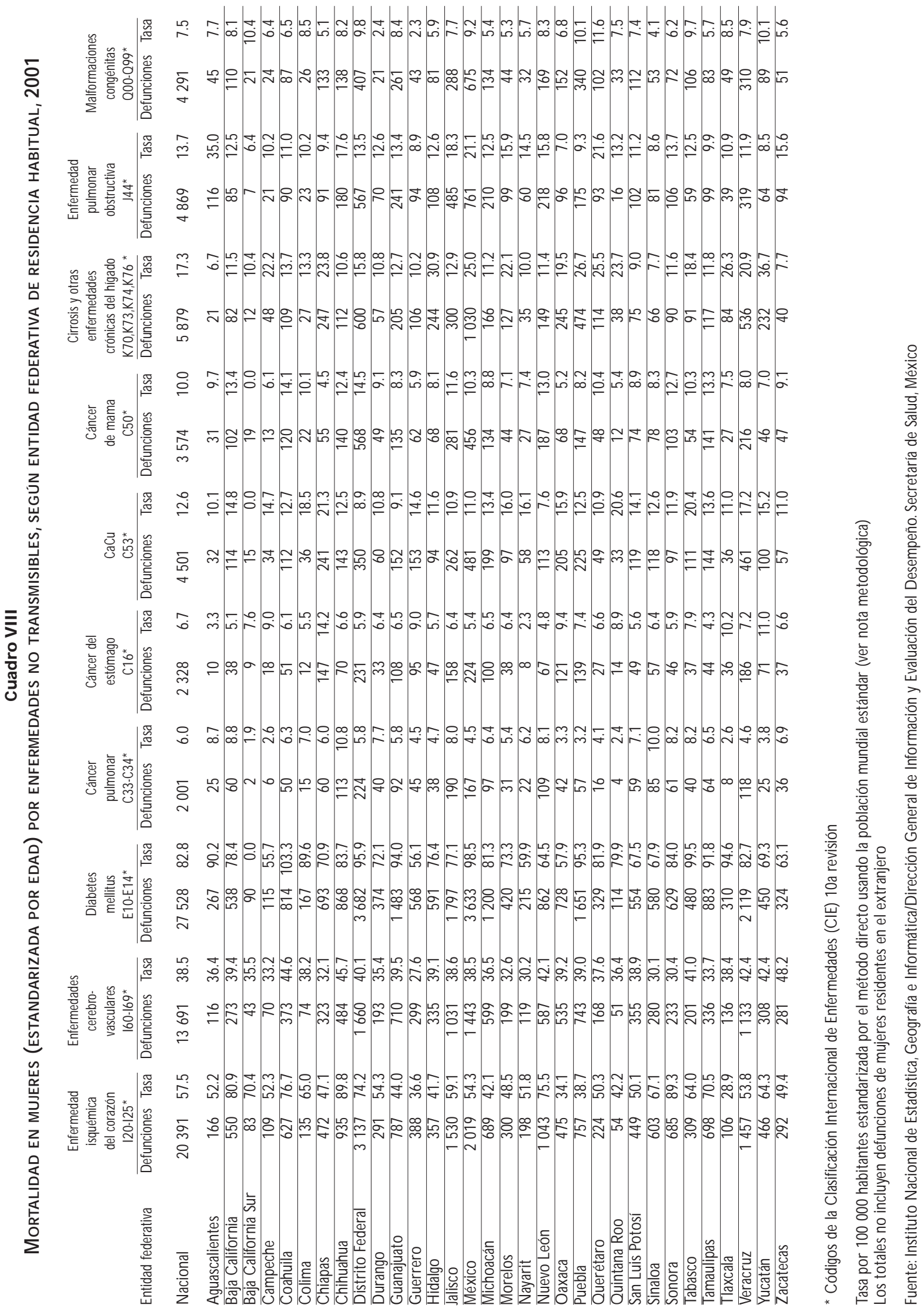


\section{Cuadro IX \\ Mortalidad en MUjeRes (estandarizada POR EDAD) POR LESIONES, SEgún ENTIDAD FEDerativa DE RESIDENCIA HABITUAL, 2001}

\begin{tabular}{|c|c|c|c|c|c|c|c|c|c|c|c|c|}
\hline Entidad federativa & \multicolumn{2}{|c|}{$\begin{array}{c}\text { Total } \\
\text { lesiones } \\
\text { V01-Y98* } \\
\text { Defunciones }\end{array}$} & \multicolumn{2}{|c|}{$\begin{array}{c}\text { Accidentes de } \\
\text { tráfico vehículos } \\
\text { de motor }\end{array}$} & \multicolumn{2}{|c|}{$\begin{array}{c}\text { Los } \\
\text { demás } \\
\text { accidentes } \\
\text { Defunciones Tasa }\end{array}$} & \multicolumn{2}{|c|}{$\begin{array}{c}\text { Homicidios } \\
\text { X85-Y09* } \\
\text { Defunciones Tasa }\end{array}$} & \multicolumn{2}{|c|}{$\begin{array}{c}\text { Suicidios } \\
\times 60-X 84 * \\
\text { Defunciones Tasa }\end{array}$} & \multicolumn{2}{|c|}{$\begin{array}{c}\text { Las demás } \\
\text { lesiones } \\
\text { Y10-Y98* } \\
\text { Defunciones Tasa }\end{array}$} \\
\hline N acional & 10626 & 23.3 & 3053 & 6.8 & 5221 & 11.7 & 1263 & 2.6 & 674 & 1.3 & 700 & 1.6 \\
\hline A guascalientes & 92 & 22.5 & 35 & 8.6 & 47 & 11.8 & 5 & 1.1 & 5 & 1.0 & 8 & 1.8 \\
\hline Baja California & 348 & 31.2 & 41 & 3.6 & 245 & 22.5 & 41 & 3.3 & 14 & 1.1 & 12 & 1.3 \\
\hline Baja C alifornia Sur & 38 & 18.9 & 21 & 9.9 & 10 & 6.0 & 5 & 2.2 & 2 & 0.8 & 2 & 0.0 \\
\hline Campeche & 67 & 22.0 & 20 & 6.7 & 25 & 8.1 & 4 & 1.9 & 16 & 4.8 & 4 & 1.3 \\
\hline Coahuila & 230 & 22.0 & 53 & 5.3 & 127 & 12.6 & 31 & 2.6 & 18 & 1.4 & 10 & 1.1 \\
\hline Colima & 67 & 26.1 & 22 & 9.4 & 29 & 11.5 & 7 & 2.4 & 5 & 1.5 & 5 & 1.7 \\
\hline Chiapas & 339 & 20.1 & 25 & 1.1 & 283 & 17.3 & 19 & 1.1 & 11 & 0.5 & 10 & 0.8 \\
\hline Chihuahua & 428 & 29.4 & 122 & 8.5 & 206 & 14.3 & 74 & 4.8 & 26 & 1.7 & 3 & 0.3 \\
\hline Distrito Federal & 945 & 21.6 & 357 & 8.3 & 349 & 8.2 & 102 & 2.2 & 72 & 1.5 & 115 & 2.6 \\
\hline Durango & 132 & 18.7 & 25 & 3.6 & 81 & 11.9 & 14 & 1.6 & 11 & 1.3 & 3 & 0.5 \\
\hline Guanajuato & 531 & 23.3 & 185 & 7.8 & 257 & 11.8 & 41 & 1.7 & 36 & 1.5 & 28 & 1.3 \\
\hline Guerrero & 356 & 26.1 & 85 & 6.6 & 188 & 13.7 & 60 & 4.4 & 17 & 1.0 & 16 & 1.1 \\
\hline Hidalgo & 270 & 24.7 & 63 & 6.0 & 156 & 14.2 & 20 & 1.8 & 14 & 1.3 & 20 & 1.7 \\
\hline Jalisco & 773 & 24.8 & 314 & 10.4 & 342 & 10.8 & 48 & 1.4 & 42 & 1.3 & 44 & 1.3 \\
\hline México & 1389 & 24.5 & 452 & 8.0 & 504 & 9.5 & 333 & 5.4 & 69 & 1.0 & 63 & 1.3 \\
\hline Michoacán & 545 & 27.4 & 148 & 7.7 & 249 & 12.4 & 73 & 3.7 & 40 & 1.9 & 48 & 2.4 \\
\hline Morelos & 191 & 26.3 & 48 & 7.0 & 91 & 12.6 & 30 & 4.1 & 11 & 1.2 & 23 & 3.1 \\
\hline $\mathrm{N}$ ayarit & 100 & 22.2 & 28 & 6.3 & 46 & 10.2 & 16 & 3.2 & 7 & 1.9 & 5 & 1.2 \\
\hline N uevo León & 341 & 19.4 & 33 & 1.8 & 275 & 16.0 & 9 & 0.4 & 19 & 1.0 & 12 & 0.8 \\
\hline 0 axaca & 378 & 23.3 & 108 & 7.0 & 181 & 10.9 & 59 & 3.7 & 17 & 0.9 & 24 & 1.4 \\
\hline Puebla & 552 & 23.1 & 155 & 6.8 & 289 & 11.9 & 57 & 2.5 & 32 & 1.1 & 27 & 1.2 \\
\hline Q uerétaro & 173 & 25.6 & 68 & 10.3 & 69 & 10.6 & 19 & 2.3 & 13 & 1.7 & 6 & 1.0 \\
\hline Q uintana Roo & 92 & 28.0 & 29 & 6.9 & 38 & 15.9 & 15 & 3.1 & 5 & 1.2 & 5 & 1.0 \\
\hline San Luis Potosí & 208 & 18.8 & 50 & 4.4 & 104 & 9.6 & 28 & 2.5 & 24 & 2.0 & 9 & 1.0 \\
\hline Sinaloa & 246 & 22.1 & 65 & 5.6 & 142 & 13.2 & 23 & 2.0 & 7 & 0.6 & 22 & 2.1 \\
\hline Sonora & 236 & 23.0 & 89 & 8.5 & 107 & 10.9 & 19 & 1.6 & 20 & 1.9 & 7 & 0.9 \\
\hline Tabasco & 205 & 25.0 & 46 & 5.3 & 126 & 16.4 & 10 & 0.9 & 20 & 2.0 & 9 & 1.4 \\
\hline Tamaulipas & 274 & 21.6 & 98 & 7.4 & 122 & 10.2 & 26 & 1.8 & 16 & 1.2 & 14 & 1.2 \\
\hline Tlaxcala & 97 & 21.6 & 23 & 5.6 & 55 & 12.2 & 13 & 2.8 & 5 & 0.9 & 4 & 1.0 \\
\hline Veracruz & 669 & 20.2 & 146 & 4.5 & 314 & 9.6 & 52 & 1.6 & 45 & 1.2 & 123 & 3.6 \\
\hline Yucatán & 167 & 21.3 & 36 & 4.7 & 99 & 12.7 & 2 & 0.2 & 24 & 2.8 & 19 & 2.6 \\
\hline Zacatecas & 147 & 21.1 & 63 & 9.9 & 65 & 8.8 & 8 & 1.1 & 11 & 1.4 & 0 & 0 \\
\hline
\end{tabular}

* Códigos de la Clasificación Internacional de Enfermedades (CIE) 10a revisión

₹ Códigos CIE 10:V02-V04 (.1, .9),V09.2-V09.3,V09.9,V12-V14 (.3-.9),V19.4-V19.6,V20-V28 (.3-.9),V29-V79 (.4-.9), V80.3-V80.5,V81.1,V82.1,V83-V86 $(.0-.3), \mathrm{V} 87.0-\mathrm{V} 87.8, \mathrm{~V} 89.2, \mathrm{~V} 89.9, \mathrm{Y} 85.0$

Tasa por 100000 habitantes estandarizada por el método directo usando la población mundial estándar (ver nota metodológica) Los totales no incluyen defunciones de mujeres residentes en el extranjero

Fuente: Instituto Nacional de Estadística, Geografía e Informática/D irección General de Información y Evaluación del Desempeño. Secretaría de Salud, México 


\section{Cuadro X}

Mortalidad en hombres (eSTANDARIZADA POR EDAD) POR ENFERMEDADES TRANSMISIBLES, PERINATALES Y DefiCIENCIAS DE LA NUTRICIÓN, SEGÚN ENTIDAD FEDERATIVA DE RESIDENCIA HABITUAL, 2001

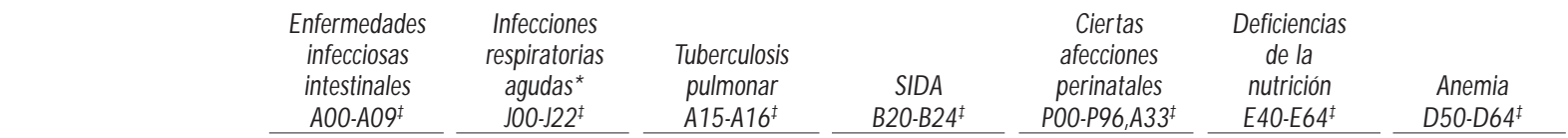

Entidad federativa $\quad \overline{\text { Defunciones Tasa }} \overline{\text { Defunciones Tasa }} \overline{\text { Defunciones Tasa }} \overline{\text { Defunciones Tasa }} \overline{\text { Defunciones Tasa }} \overline{\text { Defunciones Tasa }} \overline{\text { Defunciones Tasa }}$

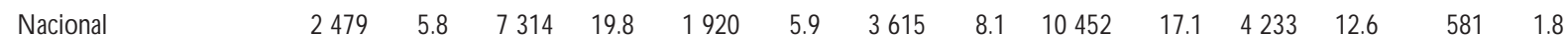

\begin{tabular}{|c|c|c|c|c|c|c|c|c|c|c|c|c|c|c|}
\hline A guascalientes & 17 & 4.6 & 26 & 8.4 & 8 & 2.0 & 27 & 6.3 & 111 & 16.4 & 44 & 14.6 & 6 & 2.0 \\
\hline Baja California & 31 & 3.1 & 189 & 21.6 & 133 & 14.9 & 166 & 13.6 & 292 & 21.0 & 46 & 7.0 & 4 & 0.7 \\
\hline Baja California Sur & 10 & 6.0 & 34 & 26.0 & 11 & 7.1 & 17 & 7.9 & 60 & 26.8 & 15 & 12.7 & 1 & 0.7 \\
\hline Campeche & 8 & 3.8 & 27 & 12.6 & 5 & 2.1 & 24 & 7.3 & 54 & 12.7 & 27 & 13.3 & 7 & 3.8 \\
\hline Coahuila & 45 & 4.9 & 85 & 9.1 & 54 & 6.6 & 51 & 4.2 & 168 & 11.5 & 77 & 10.2 & 3 & 0.4 \\
\hline Colima & 11 & 4.4 & 27 & 13.8 & 10 & 4.7 & 18 & 7.2 & 45 & 14.7 & 25 & 12.2 & 3 & 1.5 \\
\hline Chiapas & 315 & 18.9 & 372 & 29.9 & 173 & 14.8 & 123 & 7.3 & 483 & 16.4 & 178 & 15.6 & 44 & 4.2 \\
\hline Chihuahua & 61 & 4.6 & 298 & 26.8 & 67 & 6.2 & 117 & 8.0 & 396 & 22.4 & 104 & 9.8 & 8 & 0.8 \\
\hline Distrito Federal & 123 & 3.5 & 834 & 24.7 & 66 & 2.0 & 529 & 12.6 & 870 & 20.6 & 222 & 6.7 & 20 & 0.6 \\
\hline Durango & 18 & 3.1 & 93 & 18.4 & 34 & 7.1 & 25 & 3.9 & 28 & 2.8 & 39 & 8.0 & 6 & 1.1 \\
\hline Guanajuato & 135 & 5.7 & 316 & 17.0 & 37 & 2.5 & 84 & 4.4 & 619 & 18.5 & 285 & 16.2 & 44 & 2.8 \\
\hline Guerrero & 93 & 7.3 & 118 & 10.7 & 91 & 9.3 & 135 & 10.0 & 148 & 6.6 & 123 & 11.1 & 18 & 1.6 \\
\hline Hidalgo & 34 & 3.1 & 138 & 16.2 & 31 & 4.0 & 43 & 4.3 & 206 & 13.7 & 100 & 12.4 & 14 & 1.7 \\
\hline Jalisco & 96 & 3.1 & 472 & 19.1 & 91 & 4.2 & 292 & 10.9 & 677 & 16.6 & 285 & 11.4 & 19 & 0.8 \\
\hline México & 369 & 6.9 & 1287 & 27.9 & 122 & 3.5 & 364 & 5.8 & 1929 & 24.9 & 485 & 13.7 & 31 & 0.8 \\
\hline Michoacán & 75 & 3.8 & 245 & 14.1 & 32 & 2.3 & 80 & 5.0 & 361 & 12.6 & 171 & 10.9 & 32 & 2.2 \\
\hline Morelos & 28 & 4.3 & 66 & 11.7 & 25 & 5.0 & 70 & 9.6 & 130 & 14.7 & 83 & 15.4 & 15 & 2.9 \\
\hline $\mathrm{N}$ ayarit & 19 & 4.4 & 51 & 13.6 & 25 & 6.9 & 39 & 9.2 & 47 & 8.2 & 44 & 11.1 & 8 & 1.9 \\
\hline Nuevo León & 35 & 2.5 & 213 & 16.5 & 84 & 6.1 & 145 & 7.8 & 264 & 12.4 & 68 & 5.6 & 1 & 0.1 \\
\hline 0 axaca & 220 & 13.9 & 331 & 22.9 & 124 & 10.3 & 117 & 7.6 & 318 & 13.1 & 316 & 23.6 & 48 & 3.7 \\
\hline Puebla & 238 & 9.6 & 561 & 25.6 & 92 & 6.0 & 161 & 7.5 & 995 & 26.8 & 359 & 19.7 & 63 & 3.5 \\
\hline Q uerétaro & 34 & 5.7 & 73 & 13.9 & 22 & 5.9 & 25 & 4.3 & 180 & 19.2 & 70 & 17.4 & 6 & 1.4 \\
\hline Q uintana Roo & 15 & 4.3 & 32 & 22.3 & 17 & 9.5 & 54 & 13.1 & 112 & 20.7 & 21 & 13.4 & 2 & 1.8 \\
\hline San Luis Potosí & 66 & 6.2 & 158 & 16.6 & 53 & 6.7 & 42 & 4.0 & 210 & 12.5 & 113 & 12.4 & 15 & 1.9 \\
\hline Sinaloa & 23 & 2.4 & 98 & 11.0 & 64 & 7.4 & 75 & 6.2 & 56 & 4.2 & 76 & 8.3 & 4 & 0.5 \\
\hline Sonora & 47 & 4.9 & 134 & 16.1 & 54 & 6.6 & 53 & 5.0 & 202 & 16.5 & 124 & 16.2 & 10 & 1.3 \\
\hline Tabasco & 22 & 2.2 & 85 & 13.5 & 36 & 6.5 & 84 & 9.4 & 235 & 18.2 & 75 & 14.7 & 23 & 4.3 \\
\hline Tamaulipas & 28 & 2.3 & 176 & 18.0 & 69 & 6.9 & 89 & 6.7 & 184 & 12.1 & 71 & 7.4 & 5 & 0.6 \\
\hline Tlaxcala & 24 & 5.2 & 124 & 31.3 & 6 & 2.1 & 20 & 4.2 & 155 & 25.3 & 69 & 18.9 & 7 & 2.0 \\
\hline Veracruz & 169 & 5.2 & 405 & 14.7 & 233 & 9.4 & 459 & 14.2 & 615 & 15.0 & 391 & 15.5 & 86 & 3.4 \\
\hline Yucatán & 55 & 7.9 & 143 & 21.2 & 36 & 6.1 & 76 & 10.0 & 171 & 18.6 & 78 & 11.8 & 16 & 2.4 \\
\hline Zacatecas & 15 & 2.1 & 103 & 16.5 & 15 & 3.3 & 11 & 2.0 & 131 & 13.7 & 49 & 7.9 & 12 & 2.0 \\
\hline
\end{tabular}

* Incluye neumonía e influenza

₹ Códigos de la Clasificación Internacional de Enfermedades (CIE) 10a revisión

Tasa por 100000 habitantes estandarizada por el método directo usando la población mundial estándar (ver nota metodológica) Los totales no incluyen defunciones de hombres residentes en el extranjero

Fuente: Instituto Nacional de Estadística, Geografía e Informática/Dirección General de Información y Evaluación del Desempeño. Secretaría de Salud, México 


\section{Cuadro XI \\ MORTALIDAd EN HOMBRES (ESTANDARIZADA POR EDAD) POR ENFERMEDADES CRÓNICAS, SEgÚN ENTIDAD FEDERATIVA DE RESIDENCIA HABITUAL DEL FALLECIDO, 2001}

\begin{tabular}{|c|c|c|c|c|c|c|c|c|c|}
\hline $\begin{array}{l}\text { Entidad } \\
\text { federativa }\end{array}$ & $\begin{array}{l}\text { Enfermedad } \\
\text { Isquémica } \\
\text { del corazón } \\
\text { 120-125* }\end{array}$ & $\begin{array}{c}\text { Enfermedades } \\
\text { cerebro- } \\
\text { vasculares } \\
\text { 160-169* }\end{array}$ & $\begin{array}{c}\text { Diabetes } \\
\text { mellitus } \\
\text { E10-E14* }\end{array}$ & $\begin{array}{c}\text { Cáncer } \\
\text { pulmonar } \\
\text { C33-C34* }\end{array}$ & $\begin{array}{c}\text { Cáncer del } \\
\text { estómago } \\
\text { C16* }\end{array}$ & $\begin{array}{c}\text { Cáncer de } \\
\text { prostata } \\
\text { C61* }\end{array}$ & $\begin{array}{l}\text { Cirrosis y otras } \\
\text { enfermedades cró- } \\
\text { nicas del hígado } \\
\text { K70,K73, } \\
\text { K74,K76* }\end{array}$ & $\begin{array}{l}\text { Enfermedad } \\
\text { pulmonar } \\
\text { obstructiva } \\
\text { J44* }\end{array}$ & $\begin{array}{c}\text { Malformaciones } \\
\text { congénitas } \\
\text { Q00-Q99* }\end{array}$ \\
\hline
\end{tabular}

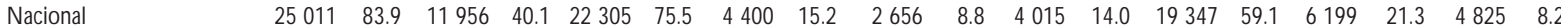

\begin{tabular}{|c|c|c|c|c|c|c|c|c|c|c|c|c|c|c|c|c|c|c|}
\hline Aguascalientes & 163 & 62.8 & 121 & 45.0 & 200 & 76.8 & 40 & 16.3 & 22 & 8.4 & 36 & 14.5 & 107 & 36.6 & 125 & 49.5 & 52 & 8.0 \\
\hline Baja California & 910 & 140.5 & 295 & 45.9 & 516 & 79.3 & 152 & 23.9 & 58 & 8.8 & 70 & 11.9 & 414 & 52.7 & 109 & 18.4 & 148 & 10.9 \\
\hline Baja C alifornia Sur & 157 & 134.1 & 34 & 29.7 & 100 & 0.0 & 38 & 36.1 & 17 & 14.4 & 15 & 0.0 & 48 & 34.0 & 29 & 26.6 & 22 & 9.7 \\
\hline Campeche & 142 & 70.4 & 94 & 46.5 & 102 & 49.2 & 23 & 11.5 & 21 & 10.7 & 18 & 9.2 & 147 & 62.6 & 35 & 18.2 & 34 & 8.2 \\
\hline Coahuila & 867 & 119.2 & 327 & 44.4 & 663 & 90.1 & 167 & 23.2 & 58 & 7.6 & 91 & 13.0 & 318 & 39.2 & 184 & 25.7 & 82 & 5.7 \\
\hline Colima & 188 & 103.0 & 54 & 30.2 & 112 & 65.3 & 32 & 18.5 & 15 & 9.1 & 22 & 12.4 & 100 & 52.9 & 44 & 23.9 & 24 & 7.7 \\
\hline Chiapas & 569 & 60.6 & 327 & 35.0 & 421 & 42.5 & 93 & 10.0 & 146 & 14.9 & 126 & 14.7 & 668 & 59.1 & 95 & 11.0 & 169 & 6.2 \\
\hline Chihuahua & 1244 & 131.2 & 427 & 45.2 & 631 & 66.0 & 225 & 24.1 & 82 & 8.6 & 140 & 15.5 & 501 & 45.9 & 289 & 31.7 & 158 & 9.2 \\
\hline Distrito Federal & 3081 & 101.6 & 1120 & 37.0 & 3050 & 102.1 & 357 & 12.2 & 277 & 9.1 & 442 & 15.0 & 1694 & 52.0 & 600 & 20.0 & 429 & 10.2 \\
\hline Durango & 348 & 73.2 & 158 & 33.0 & 321 & 68.1 & 90 & 19.3 & 37 & 8.1 & 80 & 17.3 & 150 & 29.8 & 141 & 29.2 & 31 & 3.5 \\
\hline Guanajuato & 970 & 67.5 & 555 & 38.6 & 1158 & 85.3 & 138 & 10.1 & 112 & 7.9 & 193 & 13.6 & 759 & 50.1 & 332 & 22.6 & 274 & 8.9 \\
\hline Guerrero & 497 & 54.0 & 266 & 29.2 & 453 & 50.1 & 95 & 10.7 & 90 & 9.7 & 109 & 12.2 & 374 & 40.0 & 133 & 14.7 & 61 & 3.0 \\
\hline Hidalgo & 374 & 51.9 & 275 & 38.2 & 452 & 64.5 & 61 & 8.6 & 56 & 8.1 & 90 & 13.1 & 738 & 99.6 & 98 & 13.8 & 120 & 8.4 \\
\hline Jalisco & 1757 & 83.9 & 823 & 38.4 & 1570 & 78.6 & 364 & 18.4 & 176 & 8.5 & 330 & 15.8 & 1108 & 50.6 & 549 & 25.5 & 340 & 9.0 \\
\hline México & 2297 & 74.1 & 1174 & 38.0 & 3134 & 95.8 & 308 & 9.8 & 229 & 6.7 & 360 & 12.9 & 3290 & 83.8 & 770 & 27.6 & 807 & 10.6 \\
\hline Michoacán & 898 & 64.7 & 508 & 35.8 & 899 & 68.8 & 211 & 15.7 & 123 & 9.2 & 227 & 16.2 & 604 & 44.5 & 299 & 20.7 & 147 & 5.9 \\
\hline Morelos & 365 & 71.6 & 169 & 33.4 & 350 & 69.3 & 55 & 11.0 & 49 & 9.8 & 64 & 12.9 & 314 & 56.4 & 125 & 25.0 & 64 & 7.5 \\
\hline $\mathrm{N}$ ayarit & 273 & 78.5 & 124 & 35.4 & 157 & 47.7 & 80 & 24.6 & 25 & 7.5 & 55 & 15.4 & 87 & 25.3 & 99 & 28.4 & 38 & 6.6 \\
\hline Nuevo León & 1519 & 127.3 & 551 & 46.7 & 681 & 57.3 & 308 & 26.6 & 76 & 6.2 & 172 & 15.2 & 410 & 31.0 & 279 & 24.8 & 178 & 8.5 \\
\hline 0 axaca & 514 & 43.8 & 430 & 36.7 & 639 & 56.6 & 61 & 5.5 & 106 & 9.1 & 110 & 9.3 & 945 & 80.9 & 91 & 7.7 & 158 & 6.8 \\
\hline Puebla & 810 & 53.8 & 658 & 43.6 & 1335 & 91.2 & 109 & 7.4 & 117 & 7.9 & 159 & 10.8 & 1800 & 116.0 & 182 & 12.1 & 363 & 10.2 \\
\hline Q uerétaro & 283 & 77.4 & 129 & 35.0 & 259 & 71.9 & 28 & 8.5 & 22 & 6.6 & 50 & 15.2 & 452 & 105.9 & 92 & 26.7 & 88 & 10.3 \\
\hline Q uintana Roo & 133 & 90.7 & 55 & 36.1 & 88 & 56.4 & 20 & 14.0 & 27 & 16.8 & 13 & 11.0 & 141 & 65.3 & 27 & 23.2 & 33 & 6.1 \\
\hline San Luis Potosí & 489 & 63.1 & 344 & 44.3 & 442 & 58.5 & 87 & 11.6 & 68 & 8.7 & 123 & 16.1 & 322 & 41.0 & 176 & 22.2 & 121 & 7.6 \\
\hline Sinaloa & 802 & 96.8 & 313 & 37.5 & 513 & 63.3 & 309 & 38.8 & 75 & 8.9 & 118 & 14.1 & 190 & 21.6 & 200 & 24.4 & 59 & 4.5 \\
\hline Sonora & 1036 & 140.5 & 286 & 39.3 & 527 & 71.4 & 223 & 31.0 & 98 & 13.1 & 133 & 19.0 & 256 & 31.0 & 184 & 25.9 & 87 & 7.0 \\
\hline Tabasco & 401 & 89.0 & 224 & 50.9 & 370 & 79.3 & 72 & 15.5 & 49 & 10.8 & 72 & 17.4 & 233 & 42.9 & 84 & 19.5 & 123 & 10.3 \\
\hline Tamaulipas & 1060 & 120.0 & 368 & 42.0 & 691 & 78.5 & 223 & 25.8 & 96 & 11.0 & 94 & 11.2 & 323 & 33.8 & 192 & 22.8 & 76 & 5.1 \\
\hline Tlaxcala & 124 & 40.7 & 130 & 42.7 & 267 & 91.8 & 9 & 2.9 & 12 & 4.3 & 28 & 9.2 & 220 & 71.2 & 48 & 15.0 & 70 & 11.8 \\
\hline Veracruz & 1766 & 76.7 & 1110 & 47.9 & 1681 & 72.9 & 248 & 11.0 & 211 & 9.1 & 348 & 15.6 & 2118 & 85.5 & 354 & 15.8 & 319 & 8.1 \\
\hline Yucatán & 609 & 97.4 & 311 & 50.3 & 264 & 45.3 & 80 & 13.6 & 66 & 11.2 & 59 & 10.0 & 387 & 63.1 & 79 & 12.8 & 85 & 9.2 \\
\hline Zacatecas & 365 & 72.6 & 196 & 39.4 & 259 & 55.6 & 94 & 20.0 & 40 & 8.4 & 68 & 13.5 & 129 & 27.4 & 155 & 30.3 & 65 & 7.6 \\
\hline
\end{tabular}

* Códigos de la Clasificación Internacional de Enfermedades (CIE) 10a revisión

Tasa por 100000 habitantes estandarizada por el método directo usando la población mundial estándar (ver nota metodológica) Los totales no incluyen defunciones de hombres residentes en el extranjero

Fuente: Instituto Nacional de Estadística, Geografía e Informática/Dirección General de Información y Evaluación del Desempeño. Secretaría de Salud, México 


\section{Cuadro XII \\ Mortalidad en hombres (eStANDARIZAda POR eDAD) POR LeSIONES, SEgúN ENTIDAD federativa DE RESIDENCIA HABITUAL DEL FALLECIDO, 2001}

\begin{tabular}{|c|c|c|c|c|c|c|c|c|c|c|c|c|}
\hline \multirow[b]{2}{*}{ Entidad federativa } & \multicolumn{2}{|c|}{$\begin{array}{c}\text { Total lesiones } \\
\text { V01-Y98* }\end{array}$} & \multicolumn{2}{|c|}{$\begin{array}{c}\text { Accidentes } \\
\text { de tráfico } \\
\text { vehículos de motor }\end{array}$} & \multicolumn{2}{|c|}{$\begin{array}{l}\text { Los demás } \\
\text { accidentes }\end{array}$} & \multicolumn{2}{|c|}{$\begin{array}{l}\text { Homicidios } \\
\text { X85-Y09* }\end{array}$} & \multicolumn{2}{|c|}{$\begin{array}{c}\text { Suicidios } \\
\times 60-\times 84^{*}\end{array}$} & \multicolumn{2}{|c|}{$\begin{array}{l}\text { Las demás lesiones } \\
\text { intencionales } \\
\text { Y10-Y98* }\end{array}$} \\
\hline & Defunciones & Tasa & Defunciones & Tasa & Defunciones & Tasa & Defunciones & Tasa & Defunciones & Tasa & Defunciones & Tasa \\
\hline$N$ acional & 40504 & 93.5 & 10697 & 24.5 & 15857 & 37.9 & 8885 & 19.5 & 3110 & 6.9 & 2194 & 5.3 \\
\hline A guascalientes & 315 & 76.5 & 149 & 35.5 & 102 & 26.0 & 20 & 5.0 & 41 & 9.3 & 6 & 1.9 \\
\hline Baja California & 1403 & 124.3 & 179 & 16.6 & 749 & 68.9 & 345 & 28.0 & 83 & 6.7 & 49 & 4.3 \\
\hline Baja C alifornia Sur & 182 & 97.5 & 76 & 38.6 & 67 & 38.3 & 15 & 6.7 & 21 & 12.5 & 5 & 0.4 \\
\hline Campeche & 255 & 81.9 & 53 & 17.5 & 92 & 31.4 & 46 & 14.4 & 54 & 15.7 & 13 & 3.8 \\
\hline Coahuila & 813 & 76.1 & 242 & 22.7 & 327 & 32.1 & 122 & 10.4 & 115 & 10.2 & 12 & 1.2 \\
\hline Colima & 235 & 93.8 & 62 & 24.9 & 92 & 36.2 & 51 & 22.0 & 21 & 7.7 & 10 & 3.3 \\
\hline Chiapas & 1709 & 105.2 & 95 & 5.8 & 1398 & 87.0 & 163 & 9.3 & 32 & 1.8 & 33 & 2.2 \\
\hline Chihuahua & 1917 & 131.0 & 413 & 28.3 & 745 & 53.1 & 553 & 35.4 & 202 & 13.9 & 5 & 0.5 \\
\hline Distrito Federal & 2948 & 72.7 & 845 & 21.1 & 720 & 19.4 & 753 & 16.8 & 305 & 7.2 & 352 & 8.9 \\
\hline Durango & 619 & 92.2 & 102 & 15.3 & 309 & 47.2 & 155 & 22.0 & 50 & 7.1 & 4 & 0.7 \\
\hline Guanajuato & 1714 & 82.6 & 677 & 33.0 & 643 & 32.0 & 196 & 8.9 & 132 & 5.6 & 77 & 3.8 \\
\hline Guerrero & 1490 & 122.2 & 261 & 20.7 & 569 & 48.0 & 578 & 47.0 & 59 & 4.9 & 27 & 2.0 \\
\hline Hidalgo & 883 & 91.0 & 199 & 20.1 & 469 & 49.2 & 103 & 10.6 & 51 & 4.9 & 65 & 6.6 \\
\hline Jalisco & 2714 & 96.5 & 1018 & 36.6 & 908 & 33.0 & 423 & 14.2 & 248 & 8.4 & 143 & 5.3 \\
\hline México & 4830 & 86.5 & 1402 & 25.1 & 1397 & 27.6 & 1687 & 28.0 & 205 & 3.1 & 185 & 4.0 \\
\hline Michoacán & 2113 & 119.3 & 553 & 31.2 & 703 & 40.0 & 568 & 32.1 & 127 & 6.7 & 171 & 9.9 \\
\hline Morelos & 629 & 90.4 & 152 & 22.4 & 200 & 29.8 & 186 & 25.6 & 34 & 4.6 & 63 & 9.2 \\
\hline$N$ ayarit & 461 & 105.5 & 143 & 33.1 & 170 & 38.5 & 101 & 23.0 & 33 & 7.8 & 15 & 3.4 \\
\hline N uevo León & 1206 & 67.7 & 136 & 7.0 & 864 & 49.1 & 79 & 4.3 & 109 & 6.2 & 31 & 2.1 \\
\hline 0 axaca & 1704 & 118.7 & 385 & 25.8 & 613 & 43.8 & 555 & 38.6 & 74 & 4.9 & 85 & 6.2 \\
\hline Puebla & 1870 & 90.8 & 554 & 26.2 & 772 & 38.5 & 358 & 17.6 & 107 & 4.8 & 84 & 4.0 \\
\hline Q uerétaro & 656 & 112.0 & 258 & 40.9 & 238 & 44.8 & 85 & 14.1 & 47 & 7.7 & 32 & 5.4 \\
\hline Q uintana Roo & 406 & 121.2 & 101 & 30.3 & 148 & 46.8 & 81 & 23.8 & 44 & 12.6 & 34 & 8.5 \\
\hline San Luis Potosí & 923 & 88.9 & 271 & 25.8 & 341 & 33.1 & 197 & 19.4 & 91 & 8.5 & 29 & 2.9 \\
\hline Sinaloa & 1271 & 109.0 & 360 & 31.1 & 336 & 29.9 & 470 & 38.9 & 75 & 6.6 & 37 & 3.3 \\
\hline Sonora & 1103 & 103.5 & 344 & 32.1 & 382 & 37.0 & 222 & 19.3 & 150 & 14.6 & 8 & 0.8 \\
\hline Tabasco & 1002 & 128.1 & 248 & 32.2 & 500 & 63.1 & 92 & 11.5 & 147 & 19.6 & 21 & 3.1 \\
\hline Tamaulipas & 1057 & 82.7 & 387 & 30.3 & 350 & 27.5 & 161 & 11.9 & 113 & 9.4 & 48 & 3.7 \\
\hline Tlaxcala & 391 & 90.7 & 145 & 30.6 & 154 & 39.0 & 45 & 9.7 & 20 & 4.5 & 30 & 7.8 \\
\hline Veracruz & 2553 & 83.5 & 504 & 16.0 & 1026 & 34.3 & 360 & 11.4 & 185 & 6.0 & 490 & 16.3 \\
\hline Yucatán & 525 & 69.1 & 131 & 16.8 & 246 & 33.7 & 34 & 4.4 & 92 & 11.3 & 26 & 3.7 \\
\hline Zacatecas & 607 & 95.7 & 252 & 38.7 & 227 & 36.9 & 81 & 12.7 & 43 & 6.7 & 4 & 0.7 \\
\hline
\end{tabular}

* Códigos de la Clasificación Internacional de Enfermedades (CIE) 10a revisión

₹ Códigos CIE 10:V02-V04 (.1, .9),V09.2-V09.3,V09.9,V12-V14 (.3-.9),V19.4-V19.6,V20-V28 (.3-.9),V29-V79 (.4-.9), V80.3-V80.5,V81.1,V82.1,V83-V86 $(.0-.3), \mathrm{V} 87.0-\mathrm{V} 87.8, \mathrm{~V} 89.2, \mathrm{~V} 89.9, \mathrm{Y} 85.0$

Tasa por 100000 habitantes estandarizada por el método directo usando la población mundial estándar (ver nota metodológica) Los totales no incluyen defunciones de hombres residentes en el extranjero

Fuente: Instituto Nacional de Estadística, Geografía e Informática/Dirección General de Información y Evaluación del Desempeño. Secretaría de Salud, México 


\section{Cuadro XIII}

INDICADORES DE CALIDAD DEL REGISTRO DE DEFUNCIONES, AMBOS SEXOS. MÉXICO, 2001

\begin{tabular}{|c|c|c|c|c|c|c|}
\hline & $\begin{array}{l}\text { Porcentaje de } \\
\text { defunciones } \\
\text { de registro } \\
\text { extemporáneo* }\end{array}$ & $\begin{array}{l}\text { Porcentaje de } \\
\text { defunciones } \\
\text { certificadas } \\
\text { por médico }\end{array}$ & $\begin{array}{l}\text { Porcentaje de } \\
\text { defunciones } \\
\text { certificadas por } \\
\text { médico tratante }\end{array}$ & $\begin{array}{l}\text { Porcentaje de } \\
\text { defunciones } \\
\text { ocurridas en } \\
\text { unidad médica }\end{array}$ & $\begin{array}{c}\text { Porcentaje de } \\
\text { defunciones en las } \\
\text { que no se especifica } \\
\text { la edads }\end{array}$ & $\begin{array}{c}\text { Porcentaje de } \\
\text { defunciones que } \\
\text { no se especifica } \\
\text { la derechohabiencia }\end{array}$ \\
\hline Estados Unidos Mexicanos & 2.1 & 98.0 & 32.4 & 45.1 & 0.6 & 7.8 \\
\hline Aguascalientes & 1.2 & 98.0 & 40.0 & 55.0 & 0.8 & 3.0 \\
\hline Baja California & 1.5 & 99.9 & 29.5 & 60.5 & 0.9 & 12.6 \\
\hline Baja C alifornia Sur & 0.7 & 99.6 & 30.5 & 59.0 & 0.8 & 3.0 \\
\hline Campeche & 1.1 & 98.6 & 33.0 & 48.6 & 1.8 & 6.4 \\
\hline Coahuila & 4.1 & 99.2 & 29.4 & 57.1 & 0.5 & 7.5 \\
\hline Colima & 0.6 & 99.0 & 28.6 & 52.0 & 4.6 & 5.2 \\
\hline Chiapas & 0.8 & 88.5 & 17.8 & 27.5 & 1.2 & 18.5 \\
\hline Chihuahua & 6.4 & 95.8 & 23.8 & 54.1 & 1.2 & 23.5 \\
\hline Distrito Federal & 0.5 & 99.7 & 25.9 & 54.7 & 0.1 & 3.3 \\
\hline Durango & 11.5 & 92.0 & 27.9 & 46.0 & 0.8 & 17.8 \\
\hline Guanajuato & 0.8 & 99.6 & 44.3 & 40.3 & 1.2 & 7.3 \\
\hline Guerrero & 9.6 & 99.2 & 25.1 & 34.3 & 0.4 & 14.7 \\
\hline Hidalgo & 2.5 & 98.8 & 37.1 & 38.6 & 0.7 & 8.0 \\
\hline Jalisco & 0.7 & 99.5 & 38.9 & 50.4 & 0.5 & 8.2 \\
\hline México & 0.8 & 99.8 & 30.3 & 46.5 & 0.2 & 3.8 \\
\hline Michoacán & 3.4 & 98.9 & 50.1 & 35.1 & 0.5 & 7.6 \\
\hline Morelos & 2.1 & 99.7 & 36.2 & 45.2 & 1.0 & 4.0 \\
\hline N ayarit & 6.0 & 98.1 & 28.9 & 47.1 & 0.5 & 7.2 \\
\hline N uevo León & 2.8 & 99.4 & 33.2 & 61.0 & 0.9 & 6.2 \\
\hline 0 axaca & 0.8 & 89.9 & 29.2 & 23.4 & 0.6 & 8.9 \\
\hline Puebla & 1.0 & 93.9 & 41.1 & 32.6 & 0.3 & 9.6 \\
\hline Q uerétaro & 1.3 & 98.9 & 32.4 & 44.6 & 0.7 & 3.4 \\
\hline Q uintana Roo & 2.1 & 99.1 & 26.5 & 53.0 & 3.8 & 11.4 \\
\hline San Luis Potosí & 2.8 & 98.5 & 35.8 & 40.6 & 0.4 & 6.0 \\
\hline Sinaloa & 4.4 & 99.1 & 26.6 & 53.9 & 0.5 & 8.2 \\
\hline Sonora & 0.9 & 98.9 & 25.5 & 58.0 & 0.2 & 8.5 \\
\hline Tabasco & 0.6 & 99.6 & 29.4 & 41.5 & 0.6 & 5.4 \\
\hline Tamaulipas & 4.9 & 98.8 & 30.0 & 55.3 & 0.8 & 5.7 \\
\hline Tlaxcala & 1.2 & 99.5 & 47.2 & 36.1 & 0.4 & 4.6 \\
\hline Veracruz & 1.8 & 98.8 & 33.1 & 36.7 & 0.7 & 6.3 \\
\hline Yucatán & 0.3 & 99.1 & 25.6 & 47.2 & 0.8 & 4.4 \\
\hline Zacatecas & 3.6 & 95.7 & 41.1 & 36.6 & 0.5 & 11.0 \\
\hline
\end{tabular}

* Se refiere a las defunciones ocuridas en años anteriores al de registro

₹ $\mathrm{N}$ o se incluyen las muertes por causas violentas

$\S$ Incluye los no especificados en años y días

Fuente: Instituto N acional de Estadística, Geografía e Informática/Dirección General de Información y Evaluación del Desempeño. Secretaría de Salud, México 


\section{Cuadro XIV}

\section{INDICADORES DE CALIDAD DEL REGISTRO DE DEFUNCIONES, AMBOS SEXOS. MÉXICO, 2001}

\begin{tabular}{|c|c|c|c|c|c|}
\hline Entidad federativa & $\begin{array}{l}\text { Mal definidas* } \\
\text { R00-R9g }\end{array}$ & $\begin{array}{c}\text { Tumores de sitio mal definido } \\
\text { C78-C80,C97 }\end{array}$ & $\begin{array}{c}\text { Mal definidas de causa externa } \\
\text { Y10-Y34 }\end{array}$ & $\begin{array}{c}\text { Insuficiencia cardíaca } \\
150^{*}\end{array}$ & $\begin{array}{c}\text { Insuficiencia hepática }{ }^{\alpha} \\
\mathrm{K} 72^{\neq}\end{array}$ \\
\hline Estados Unidos Mexicanos & 2.1 & 4.5 & 4.6 & 11.3 & 7.7 \\
\hline A guascalientes & 0.9 & 4.4 & 0.7 & 8.6 & 9.2 \\
\hline Baja California & 2.0 & 8.6 & 3.1 & 5.9 & 6.9 \\
\hline Baja C alifornia Sur & 1.0 & 6.1 & 1.4 & 7.7 & 8.7 \\
\hline Campeche & 3.4 & 4.3 & 3.7 & 9.8 & 6.7 \\
\hline Coahuila & 1.8 & 3.8 & 0.8 & 8.7 & 5.1 \\
\hline Colima & 1.4 & 2.9 & 3.6 & 6.9 & 7.2 \\
\hline Chiapas & 7.1 & 4.9 & 1.2 & 16.5 & 7.0 \\
\hline Chihuahua & 2.0 & 5.3 & 0.1 & 8.8 & 3.9 \\
\hline Distrito Federal & 0.8 & 3.7 & 10.0 & 6.1 & 13.0 \\
\hline Durango & 3.3 & 4.9 & 0.3 & 10.6 & 5.3 \\
\hline Guanajuato & 1.7 & 3.1 & 3.5 & 14.1 & 4.5 \\
\hline Guerrero & 3.4 & 5.2 & 1.6 & 13.1 & 8.1 \\
\hline Hidalgo & 1.6 & 3.8 & 6.2 & 17.6 & 8.1 \\
\hline Jalisco & 1.0 & 4.0 & 4.1 & 10.2 & 7.6 \\
\hline México & 0.5 & 4.2 & 2.7 & 10.7 & 10.1 \\
\hline Michoacán & 2.1 & 4.3 & 7.4 & 19.5 & 7.3 \\
\hline Morelos & 1.2 & 5.2 & 8.4 & 8.9 & 6.8 \\
\hline $\bar{N}$ ayarit & 1.9 & 4.2 & 2.1 & 8.3 & 6.7 \\
\hline N uevo León & 1.1 & 6.4 & 1.6 & 8.7 & 7.0 \\
\hline 0 axaca & 5.5 & 3.9 & 4.4 & 27.8 & 6.8 \\
\hline Puebla & 4.0 & 5.3 & 3.5 & 19.1 & 5.1 \\
\hline Q uerétaro & 2.0 & 4.0 & 3.9 & 11.7 & 7.1 \\
\hline Q uintana Roo & 1.6 & 3.4 & 7.6 & 9.7 & 6.1 \\
\hline San Luis Potosí & 3.2 & 5.0 & 2.2 & 15.5 & 7.0 \\
\hline Sinaloa & 2.0 & 4.6 & 2.6 & 7.3 & 7.0 \\
\hline Sonora & 1.2 & 5.3 & 0.4 & 8.3 & 7.3 \\
\hline Tabasco & 1.5 & 4.4 & 1.5 & 10.1 & 8.1 \\
\hline Tamaulipas & 1.2 & 4.0 & 4.4 & 8.4 & 6.5 \\
\hline Tlaxcala & 2.8 & 5.2 & 5.5 & 20.0 & 4.9 \\
\hline Veracruz & 2.1 & 3.9 & 18.3 & 11.9 & 6.0 \\
\hline Yucatán & 4.4 & 2.4 & 4.0 & 8.9 & 4.7 \\
\hline Zacatecas & 3.2 & 6.1 & 2.4 & 14.8 & 6.6 \\
\hline
\end{tabular}

* Porcentaje respecto al total de defunciones

₹ Porcentaje respecto al total de tumores malignos $\mathrm{C} 00-\mathrm{C} 97$

$\S$ Porcentaje respecto al total de defunciones por causa externa

* Porcentaje respecto a las enfermedades del corazón $100-151^{*}$

\& Porcentaje respecto a las enfermedades del hígado $\mathrm{K} 70^{\prime} \mathrm{K} 76^{*}$

* Códigos de la Clasificación Internacional de Enfermedades (CIE) 10a revisión

Fuente: Instituto Nacional de Estadística, Geografía e Informática/Dirección General de Información y Evaluación del Desempeño. Secretaría de Salud, México 